\title{
Probing neutrino Dirac mass in left-right symmetric models at the LHC and next generation colliders
}

\author{
Juan Carlos Helo, ${ }^{1, *}$ Haolin Li, ${ }^{2,6, \dagger}$ Nicolás A. Neill, ${ }^{3,4, \$}$ Michael Ramsey-Musolf, ${ }^{2,5, \S}$ and Juan Carlos Vasquez ${ }^{3,4, \|}$ \\ ${ }^{1}$ Departamento de Física, Facultad de Ciencias, Universidad de La Serena, La Serena 1720170, Chile \\ ${ }^{2}$ Amherst Center for Fundamental Interactions, Department of Physics, \\ University of Massachusetts, Amherst, Massachusetts 01003, U.S.A \\ ${ }^{3}$ Departamento de Fìsica Universidad Técnica Federico Santa María, Valparaíso 2340000, Chile \\ ${ }^{4}$ Centro Cientifico Tecnologico de Valparaiso, Valparaiso 2340000, Chile \\ ${ }^{5}$ Kellogg Radiation Laboratory, California Institute of Technology, Pasadena, California 91125 USA \\ ${ }^{6}$ Institute of Theoretical Physics, China Academic of Science, Beijing 100190, China
}

(Received 14 December 2018; published 27 March 2019)

\begin{abstract}
We assess the sensitivity of the LHC, its high energy upgrade, and a prospective $100 \mathrm{TeV}$ hadronic collider to the Dirac Yukawa coupling of the heavy neutrinos in left-right symmetric models (LRSMs). We focus specifically on the trilepton final state in regions of parameter space yielding prompt decays of the right-handed gauge bosons $\left(W_{R}\right)$ and neutrinos $\left(N_{R}\right)$. In the minimal LRSM, the Dirac Yukawa couplings are completely fixed in terms of the mass matrices for the heavy and light neutrinos. In this case, the trilepton signal provides a direct probe of the Dirac mass term for a fixed $W_{R}$ and $N_{R}$ mass. We find that while it is possible to discover the $W_{R}$ at the LHC, probing the Dirac Yukawa couplings will require a $100 \mathrm{TeV} p p$ collider. We also show that the observation of the trilepton signal at the LHC would indicate the presence of a nonminimal LRSM scenario.
\end{abstract}

DOI: $10.1103 /$ PhysRevD.99.055042

\section{INTRODUCTION}

Soon after the appearance of the original works [1-3], the minimal left-right symmetric model (mLRSM) has been proposed to connect the smallness of neutrino masses with the spontaneous violation of parity [4-6]. The origin of neutrino masses within the mLRSM must be understood in analogy with the explanation of the origin of mass within the standard model (SM). In the SM, fermion masses are obtained through the Higgs mechanism, for which one manifestation is the proportionality of a given Higgs boson fermionic branching ratio to the square of the corresponding fermion mass. The Higgs boson has been discovered at the LHC by the ATLAS and CMS collaborations [7], and the measured branching ratio into bottom quark and $\tau$ lepton pairs agree with the SM expectations [8].

\footnotetext{
*jchelo@userena.cl

lihaolin1991@gmail.com

\$mjrm@physics.umass.edu

\$nicolas.neill@gmail.com

"juan.vasquezcar@usm.cl
}

Published by the American Physical Society under the terms of the Creative Commons Attribution 4.0 International license. Further distribution of this work must maintain attribution to the author(s) and the published article's title, journal citation, and DOI. Funded by SCOAP.
In the neutrino sector, the situation becomes less clear since neutrinos are electrically neutral. While a SM-like pure Dirac mass is a possibility, the magnitudes of associated Yukawa couplings would be considerably smaller than for the charged elementary fermions. A theoretically attractive alternative is the seesaw mechanism [5,9-13], which exploits the possibility that the electrically neutral neutrino may be its own antiparticle. Neutrino masses in the mLRSM arise from a combination of two versions of the seesaw mechanism, the so-called Type-I, and Type-II variants.

If left-right symmetry is realized in nature, it will be important to establish whether the mLRSM is at the same level as the SM regarding the origin of fermion masses. Soon after its original proposal, approaches for probing the Yukawa couplings of heavy and light neutrinos were considered. The Yukawa sector for the light neutrinos may be in principle probed in low energy experiments, such as neutrinoless double beta decay and oscillation experiments. The Yukawa couplings of heavy neutrinos (HNs) can be probed in high energy experiments through the Keung-Senjanović (KS) process [14], which consists in the production of an on-shell, heavy $W_{R}$ gauge boson decaying into two right-handed leptons and two jets. ATLAS latest search extends the exclusion region to $M_{W_{R}}=4.7 \mathrm{TeV}$ for both Dirac and Majorana heavy neutrinos. More recently, in Refs. [15-17] it was found 
that the Dirac Yukawa coupling of neutrinos (which is proportional to the mixing between heavy and light neutrinos) can be unambiguously obtained once all light and heavy neutrino masses and mixing angles are measured. Therefore, this puts the mLRSM as a testable model of neutrino masses and calls for the experimental verification of the relation between the Dirac mass and the heavy and light neutrino masses, the main subject of this work.

It is worth emphasizing that without left-right symmetry, the connection between the Dirac mass matrix and the heavy and light neutrino mass matrices is lost. This can be explicitly seen in the Casas-Ibarra parametrization [18], where the Dirac mass matrix is given in terms of the heavy and light neutrino mass matrices up to an arbitrary complex, orthogonal matrix, whose elements are not even bounded. This situation contrasts with the mLRSM, since within this framework the imposition of a discrete LR symmetry is sufficient to fix the arbitrary orthogonal matrix in terms of the heavy and light neutrino mass matrices (see for instance Refs. [15-17]).

In the minimal setup, the Dirac Yukawa coupling of heavy neutrinos are predicted to be small [15], and for this reason all processes that probe the Dirac Yukawa couplings are known to be suppressed. Dirac Yukawa couplings could in principle be probed by considering angular asymmetries in the rare channel with one right-handed charged lepton, one left-handed charged lepton and two jets in the final state [15]. In practice, as we shall show, this may not be feasible with the statistics one expects even at the $100 \mathrm{TeV}$ machine. The reason is that for a large number of events $N$, the statistical error goes as $\sqrt{N}$ and hence the asymmetry one wishes to measure should be at least of the order of $\sqrt{N} / N=1 / \sqrt{N}$. For instance, for the mLRSM and in the best case scenario with $W_{R}$ boson mass of $6 \mathrm{TeV}$, the branching ratio of the HNs to charged leptons and one $W$ boson is of the order of $10^{-4}$. Hence, probing this small branching ratio would require at least $10^{8}$ signal events (S), which is not feasible at either the LHC with high luminosity or a next $100 \mathrm{TeV} p p$-collider. For a complete phenomenological study of the two leptons and two jets channel, see e.g., Refs. $[19,20]$.

Consequently, in this work we propose instead that the ideal channel for probing the Dirac mass term of heavy neutrinos is through the purely leptonic decay $W_{R}^{ \pm} \rightarrow l^{ \pm} N \rightarrow l^{ \pm}\left(N \rightarrow l^{ \pm} W^{\mp} \rightarrow l^{ \pm} l^{\prime \mp} \nu\right)$. This channel has been previously studied in the context of the type I seesaw extension of the SM with fermion singlets in Ref. [21] and more recently searched for by the CMS collaboration [22]. It has also been studied in the context of a left-right symmetric model with an inverse seesaw mechanism in Refs. [23,24]. What is new in this work with respect to previous studies is that first: we assumed for the minimal model, that the decay of the $\mathrm{HN}$ into one lepton and two jets (KS channel) is the dominant channel for the minimal model and second, we take into account for our analysis the connection of the Dirac Yukawa couplings with the heavy and light neutrino Yukawa couplings of Refs. [15-17]. This connection turns out to be important from both theoretical and phenomenological sides, since as we shall show, it makes the model more predictive. We would like to emphasize that this is a crucial point usually overlooked in the literature.

It provides a cleaner signal that has an advantage with respect to the KS channel since no asymmetry needs to be measured. Our main findings may be summarized as follows: in order to test the mLRSM prediction for the mixing between the heavy and light neutrinos (or equivalently the Dirac mass), one must consider a next generation collider beyond the LHC, such as a $100 \mathrm{TeV} p p$ collider. On the other hand, and if one observes evidence for this mixing at the $\mathrm{LHC}$, it would point to a nonminimal scenario within the context of LRSMs.

The discussion of our study leading to these findings is organized as follows. In Sec. II, we briefly introduce the model in both its minimal and nonminimal incarnations. Assuming charge conjugation as the left-right symmetry for the minimal case, we explain the relationship between the Dirac mass matrix in terms of the heavy and light neutrino mass matrices. This connection is crucial to obtain the sensitivity to the Dirac mass. In Sec. III, we estimate the sensitivity at the LHC, High Energy LHC (HE-LHC) and a $100 \mathrm{TeV} p p$ collider to heavy-light neutrino mixing. We compare the reaches of these various colliders to the expectations within the mLRSM and nonminimal model discussed in Sec. II. In Sec. IV and within the minimal model, we translate the sensitivity to the heavy-light neutrino mixing into a reach on the Dirac mass. Finally in Sec. VI the conclusions are given.

\section{THE LEFT-RIGHT SYMMETRIC MODEL}

\section{A. The minimal left-right symmetric model}

The minimal left-right symmetric model [1-3] was introduced in order to explain the smallness of neutrino mass in connection with the spontaneous violation of parity [4-6]. In this work we do not pursue the $\mathcal{O}(1)$ Yukawa couplings of neutrinos, that is, without special Yukawa texture [25-32] or cancellation between Type I and Type II see-saw effect [33-35]. In this case, the LR symmetry breaking scale would be very high, such that the $W_{R}$ boson and heavy neutrino will be too heavy to be produced even in a future $100 \mathrm{TeV}$ collider. Instead, we consider the minimal framework, where we have relatively small Yukawa couplings $\left(\mathcal{O}\left(10^{-6}-10^{-5}\right)\right)$ with relatively light $W_{R}$ boson mass $(\mathcal{O}(1-10) \mathrm{TeV})$ and in the reach of present and future colliders.

The gauge group and field content: The gauge group is $\mathcal{G}=S U(2)_{L} \times S U(2)_{R} \times U(1)_{B-L}$, with an additional discrete symmetry that may be generalized parity $(\mathcal{P})$ or charge conjugation $(\mathcal{C})$. The quarks and leptons are 
doublets in the following irreducible representations of the gauge group:

$$
\begin{aligned}
q_{L}=\left(\begin{array}{l}
u \\
d
\end{array}\right)_{L}:\left(2,1, \frac{1}{3}\right), & q_{R}=\left(\begin{array}{l}
u \\
d
\end{array}\right)_{R}:\left(1,2, \frac{1}{3}\right), \\
L_{L}=\left(\begin{array}{l}
\nu \\
l
\end{array}\right)_{L}:(2,1,-1), & L_{R}=\left(\begin{array}{c}
N \\
l
\end{array}\right)_{R}:(1,2,-1) .
\end{aligned}
$$

Where $N$ represents the new heavy neutrino states, whose presence explain the smallness of neutrino masses on the basis of the see-saw mechanism [5,9-13].

The Higgs sector sector of the mLRSM [5,9], consists of one bidoublet $\Phi$, in the $(2,2,0)$ representation of $\mathcal{G}$ and two scalar triplets $\Delta_{L}$ and $\Delta_{R}$, belonging to $(3,1,2)$ and $(1,3,2)$ representation respectively

$$
\begin{aligned}
\Phi & =\left(\begin{array}{ll}
\phi_{1}^{0} & \phi_{2}^{+} \\
\phi_{1}^{-} & \phi_{2}^{0}
\end{array}\right), \\
\Delta_{L, R} & =\left(\begin{array}{cc}
\delta_{L, R}^{+} / \sqrt{2} & \delta_{L, R}^{+} \\
\delta_{L, R}^{0} & -\delta_{L, R}^{+} / \sqrt{2}
\end{array}\right) .
\end{aligned}
$$

After SSB, the vev's of the Higgs fields may be written as [6]

$$
\begin{gathered}
\langle\Phi\rangle=\left(\begin{array}{cc}
v_{1} & 0 \\
0 & v_{2} e^{i \alpha}
\end{array}\right), \\
\left\langle\Delta_{R}\right\rangle=\left(\begin{array}{cc}
0 & 0 \\
v_{R} & 0
\end{array}\right), \quad\left\langle\Delta_{L}\right\rangle=\left(\begin{array}{cc}
0 & 0 \\
v_{L} e^{i \theta_{L}} & 0
\end{array}\right),
\end{gathered}
$$

where $\alpha$ and $\theta_{L}$ are called the "spontaneous" $C P$ phase and $v_{L} \ll v_{1}^{2}+v_{2}^{2} \ll v_{R}^{2}$. All the physical effects due to $\theta_{L}$ can be neglected, since this phase is always accompanied by the small $v_{L}$.

Under the discrete left-right symmetry the fields transform as follows:

$$
\begin{gathered}
\mathcal{P}:\left\{\begin{array}{l}
\mathcal{P} f_{L} \mathcal{P}^{-1}=\gamma_{0} f_{R} \\
\mathcal{P} \Phi \mathcal{P}^{-1}=\Phi^{\dagger} \\
\mathcal{P} \Delta_{(L, R)} \mathcal{P}^{-1}=-\Delta_{(R, L)}
\end{array}\right. \\
\mathcal{C}:\left\{\begin{array}{l}
\mathcal{C} f_{L} \mathcal{C}^{-1}=C\left(\bar{f}_{R}\right)^{T} \\
\mathcal{C} \Phi \mathcal{C}^{-1}=\Phi^{T} \\
\mathcal{C} \Delta_{(L, R)} \mathcal{C}^{-1}=-\Delta_{(R, L)}^{*}
\end{array}\right.
\end{gathered}
$$

where $\gamma_{\mu}(\mu=0,1,2,3$.) are the gamma matrices and $\mathcal{C}$ is the charge conjugation operator.

Lepton masses: Lepton masses are due to the following Yukawa interactions (once the Higgs fields take their v.e.v. along their neutral components)

$$
\begin{aligned}
\mathcal{L}_{Y}= & \bar{L}_{L}\left(Y_{\Phi} \Phi+\tilde{Y}_{\Phi} \tilde{\Phi}\right) L_{R}+\frac{1}{2}\left(L_{L}^{T} \operatorname{Ci\sigma }_{2} Y_{\Delta_{L}} \Delta_{L} L_{L}\right. \\
& \left.+L_{R}^{T} C_{i \sigma_{2}} Y_{\Delta_{R}} \Delta_{R} L_{R}\right)+ \text { H.c. },
\end{aligned}
$$

where $\tilde{\Phi}=\sigma_{2} \Phi^{*} \sigma_{2}, \sigma_{2}$ is the Pauli matrix and $C \equiv i \gamma_{2} \gamma_{0}$.

Invariance of the Lagrangian under the left-right symmetry requires the Yukawa couplings to satisfy

$$
\mathcal{P}:\left\{\begin{array}{l}
Y_{\Delta_{R, L}}=Y_{\Delta_{L, R}} \\
Y_{\Phi}=Y_{\Phi}^{\dagger} \\
\tilde{Y}_{\Phi}=\tilde{Y}_{\Phi}^{\dagger}
\end{array}, \quad \mathcal{C}:\left\{\begin{array}{l}
Y_{\Delta_{R, L}}=Y_{\Delta_{L, R}}^{*} \\
Y_{\Phi}=Y_{\Phi}^{T} \\
\tilde{Y}_{\Phi}=\tilde{Y}_{\Phi}^{T}
\end{array}\right.\right.
$$

Consistent with the above notation, the neutrino mass matrix of neutrinos is of the form $[5,6]$

$$
\mathcal{L}_{\nu}=\frac{1}{2}\left(\begin{array}{ll}
\nu & N^{c}
\end{array}\right)_{L}^{T} C\left(\begin{array}{ll}
M_{L} & M_{D}^{*} \\
M_{D}^{\dagger} & M_{R}
\end{array}\right)\left(\begin{array}{c}
\nu \\
N^{c}
\end{array}\right)_{L}+\text { H.c. }
$$

where $N_{L}^{c} \equiv C \bar{N}_{R}^{T}$ and $M_{L}, M_{R}$ and $M_{D}$ are $3 \times 3$ matrices given by

$$
\begin{gathered}
M_{L} \equiv Y_{\Delta_{L}} v_{L} e^{i \theta_{L}}, \\
M_{R} \equiv Y_{\Delta_{R}}^{*} v_{R}, \\
M_{D} \equiv v_{1} Y_{\Phi}+\tilde{Y}_{\Phi} v_{2} e^{-i \alpha} .
\end{gathered}
$$

After diagonalization, the light and heavy neutrino mass matrices takes the seesaw form:

$$
\begin{gathered}
M_{\nu} \simeq M_{L}-M_{D}^{*} \frac{1}{M_{N}} M_{D}^{\dagger}, \\
M_{N} \simeq M_{R} .
\end{gathered}
$$

The contributions to the light neutrino masses proportional to $M_{D}$ and $M_{L}$ are called the Type I and Type II seesaw contributions, respectively. It follows from the seesaw formula that the eigenstates corresponding to Eqs. (12) are given by

$$
\left(\begin{array}{c}
\nu^{\prime} \\
N^{\prime c}
\end{array}\right)=\left(\begin{array}{cc}
1 & \Theta \\
-\Theta^{T} & 1
\end{array}\right)\left(\begin{array}{c}
\nu \\
N^{c}
\end{array}\right),
$$

where the heavy-light neutrino mixing is given by

$$
\Theta \simeq M_{D}^{*} M_{N}^{-1} .
$$

Finally, the charged lepton mass matrix is given by

$$
M_{l}=Y_{\Phi} v_{2} e^{i \alpha}+\tilde{Y}_{\Phi} v_{1} .
$$

As usual, the mass matrices can be diagonalized by the biunitary transformations 


$$
\begin{aligned}
& M_{l}=U_{l L} \hat{M}_{l} U_{l R}^{\dagger}, \\
& M_{\nu}=U_{\nu}^{*} \hat{M}_{\nu} U_{\nu}^{\dagger}, M_{N}=U_{N}^{*} \hat{M}_{N} U_{N}^{\dagger},
\end{aligned}
$$

where $\hat{M}_{l}, \hat{M}_{\nu}$ and $\hat{M}_{N}$ are diagonal matrices with real, positive eigenvalues.

Charged gauge interactions with leptons: From the covariant derivative and in the mass eigenstates basis, the charged current Lagrangian is

$$
\begin{aligned}
\mathcal{L}_{c c}= & \frac{g}{\sqrt{2}}\left(\bar{l}_{L} V_{L} W_{L} \nu_{L}-\bar{l}_{L} \Theta_{L} W_{L} N_{L}^{c}+\bar{l}_{R} V_{R} W_{R} N_{R}\right. \\
& \left.+\bar{l}_{R} \Theta_{R} W_{R} \nu_{R}^{c}\right)+ \text { H.c. },
\end{aligned}
$$

where $N_{R} \equiv C\left(\bar{N}_{L}^{c}\right)^{T}=i \gamma_{2} \gamma_{0}\left(N_{L}^{c}\right)^{*}, \nu_{R}^{c} \equiv C\left(\bar{\nu}_{L}\right)^{T}$ and $\gamma_{0}$ and $\gamma_{2}$ are the gamma matrices and the mixing matrices $V_{L}$, $V_{R}, \Theta_{L}$ and $\Theta_{R}$ are given by

$$
\begin{array}{cc}
V_{L}=U_{l L}^{\dagger} U_{\nu}, & \Theta_{L}=U_{l L}^{\dagger} \Theta U_{N} \\
V_{R}=U_{l R}^{\dagger} U_{N}^{*}, & \Theta_{R}=U_{l R}^{\dagger} \Theta^{\dagger} U_{\nu}^{*}
\end{array}
$$

We may use the freedom of rephasing the charged lepton fields to remove three unphysical phases from $V_{L}$, which ends up having 3 mixing angles and 3 phases, namely one Dirac and two Majorana phases. Since the freedom of rephasing the charged lepton is already used for $V_{L}$, its right-handed analog - the leptonic mixing matrix $V_{R}$-is a general $3 \times 3$ unitary matrix and may be therefore parametrized by 3 mixing angles and 6 phases.

A comment regarding the mixing matrices $\Theta_{L}$ and $\Theta_{R}$ is in order: for charge conjugation as the LR symmetry, without loss of generality one can choose $U_{l L}=U_{l R}=1$, such that $V_{L}=U_{\nu}$ and $V_{R}=U_{N}^{*}$. In this case, the mixing matrices can be written in the form

$$
\Theta_{L}=\Theta V_{R}^{*}, \quad \Theta_{R}=\Theta V_{L}^{*} .
$$

For parity as the LR symmetry, it is no longer true that one can assume $U_{l L}=U_{l R}=1$. Nevertheless, since the Dirac mass matrix is Hermitian with a very good approximation, even in this case one can write

$$
\begin{aligned}
\Theta_{L} & =\Theta V_{R}^{*}\left[1+\mathcal{O}\left(\hat{M}_{l} \tan 2 \beta \sin \alpha\right)\right], \\
\Theta_{R} & =\Theta V_{L}^{*}\left[1+\mathcal{O}\left(\hat{M}_{l} \tan 2 \beta \sin \alpha\right)\right] .
\end{aligned}
$$

where the parameter $\tan 2 \beta \sin \alpha \lesssim 2 m_{b} / m_{t}$ [36,37], with $\tan \beta \equiv v_{2} / v_{1}, m_{b}$ and $m_{t}$ are the bottom and top quark masses respectively. Therefore, up to small terms of the order $\mathcal{O}\left(\hat{M}_{l} \tan 2 \beta \sin \alpha\right)$, the heavy light mixing matrices are roughly the same for both parity and charge conjugation as the LR symmetry.

Heavy-light mixing in the mLRSM: In the MLRSM, the heavy-light neutrino mixing depends on the light and the heavy neutrino mass matrices. It is known [15] that this mixing enters in the decay of the heavy neutrino into a lefthanded charged lepton and two jets [14], and one could measure the mixing by measuring the chirality [20] of the outgoing charged lepton in order to discriminate this channel from the usual channel where the heavy neutrino decays into a right-handed charged leptons and two jets. ${ }^{1}$ Instead, in this work we point out that the same mixing enters in the purely leptonic decay of the heavy neutrinos. This channel has an advantage with respect to the channel with two leptons and two jets, since no asymmetry (chirality information) needs to be measured in order to obtain the heavy-light mixing matrix elements. In addition, from the experimental perspective, the backgrounds relevant to the purely leptonic channel are cleaner. This channel has been previously studied in Refs. [21,38] including both prompt and displaced vertex for the signal in the context of the SM extended by a fermion singlet. In the latter instance, no heavy resonance $W_{R}$ is produced in the process, which makes kinematics for the final states very different with respect to the present work.

In what follows and for the sake of illustration, we consider $\mathcal{C}$ as the LR symmetry but the same conclusions hold for the case when the LR symmetry corresponds to $\mathcal{P}$. From Eq. (7) it follows that the Dirac mass term is symmetric and Eq. (12) takes the form [15]

$$
M_{\nu} \simeq Y_{\Delta_{L}} v_{L} e^{i \theta_{L}}-M_{D}^{*} \frac{1}{M_{N}} M_{D}^{*}
$$

Multiplying from the left by $M_{N}^{-1}$ one gets [39]

$$
\begin{gathered}
M_{N}^{-1} M_{\nu} \simeq M_{N}^{-1} Y_{\Delta_{L}} v_{L} e^{i \theta_{L}}-\frac{1}{M_{N}} M_{D}^{*} \frac{1}{M_{N}} M_{D}^{*}, \\
M_{N}^{-1} M_{\nu} \simeq M_{N}^{-1} M_{L}-\Theta^{2} .
\end{gathered}
$$

Hence, the mixing angle can be written in terms of the heavy and light neutrino masses as [39]

$$
\Theta=\sqrt{\epsilon-M_{N}^{-1} M_{\nu}}=M_{D}^{*} M_{N}^{-1},
$$

with $\epsilon \equiv v_{L} / v_{R}$. See Ref. [16,17] for the determination of the analogue of Eq. (26) for parity as the LR symmetry. In what follows and for the sake of simplifying the discussion, we set $v_{L}=0$, effectively assuming type I seesaw dominance for the light neutrino masses. Notice that the choice $v_{L}$ small is technically natural, as discussed originally in Ref. [6] and more recently revisited in Ref. [40]. Finally,

\footnotetext{
${ }^{1}$ The relative strength of these two channels can be seen for instance from the "phase diagram" of the heavy-light mixing in the seesaw models in Ref. [32], and it is pointed out that without special Yukawa texture the ordinary channel with heavy neutrino decay with right-handed charged current is generally much larger than the left-handed current.
} 
notice that the mixing matrix $\Theta$ (equivalently $M_{D}$ ) is a complex matrix, so that no issues arise due to the -1 factor inside the square root and the fact that $\epsilon$ is a complex quantity. In any case, this phase phase factor has no impact in our analysis below. For a discussion of its physical significance see, for instance, Ref. [39].

In the next section and using the above leptonic channel, we study the sensitivity of the LHC, HE-LHC and a $100 \mathrm{TeV} p p$ collider, to the mixing in Eq. (26) as a function of $M_{W_{R}}$ and the lightest heavy neutrino mass $m_{N}$, for benchmark values of the other heavy neutrinos. Later, from this sensitivity and using Eq. (15), one can infer the values of $M_{D}$ that can be probed at the LHC and the next generation of hadronic colliders.

\section{B. "Nonminimal" left-right symmetric model}

As explained in the previous section, in the mLRSM the gauge group is broken to the SM group through the triplet $\Delta_{R}$ and the bidoublet scalar $\Phi$. This construction generates a seesaw mass for the right-handed neutrinos from the vacuum expectation value of the $\Delta_{R}$. Here, we will consider a slightly different LR scenario, a "nonminimal" model, in which now the LR group is broken through a doublet scalar in the $(1,2,-1)$ representation of $\mathcal{G}$ [41,42]. Adding an extra vector of gauge singlet fermions $S=\left(S_{1}, S_{2}, S_{3}\right)^{T}$ to the particle content the neutrino masses will be generated now by an inverse seesaw mechanism [43,44]. The inverse seesaw scenario in the context of left-right symmetry was studied in detail in Ref. [45]. In this section, we will only review the most important results.

We work in the basis in which the charged lepton mass matrix is diagonal. The inverse seesaw neutrino mass matrix in the interaction basis for the neutral states $\mathcal{N}=$ $\left(\nu_{L}, N^{c}, S^{c}\right)^{T}$ can be written in a $3 \times 3$ notation as:

$$
\mathcal{M}=\left(\begin{array}{ccc}
0 & M_{D}^{T} & 0 \\
M_{D} & 0 & M_{N} \\
0 & M_{N}^{T} & \mu
\end{array}\right)
$$

where $M_{D}, M_{N}$, and $\mu$ denote $3 \times 3$ matrices and the submatrix $\mu$ is taken to be diagonal. Assuming the submatrices $M_{D}, M_{N}, \mu$ have mass scales arranged hierarchically, $M_{N} \gg M_{D}$, $\mu$, the light neutrino mass matrix $M_{\nu}$ can be expressed in terms of the matrices in Eq. (27) as

$$
M_{\nu} \simeq M_{D}^{T} \frac{1}{M_{N}^{T}} \mu \frac{1}{M_{N}} M_{D}
$$

Using the biunitary transformations

$$
M_{\nu}=V_{L}^{*} m_{\nu} V_{L}^{\dagger}, \quad M_{N}=V_{R} \hat{M}_{N} U_{R}^{\dagger},
$$

the mass matrix $\mathcal{M}$ can be diagonalized into

$$
\hat{\mathcal{M}}=\left(\begin{array}{ccc}
m_{\nu} & 0 & 0 \\
0 & \hat{M}_{N}^{-} & 0 \\
0 & 0 & \hat{M}_{N}^{+}
\end{array}\right) .
$$

Here $\hat{M}_{N}^{-}, \hat{M}_{N}^{+}$and $\hat{M}_{N}$ are diagonal mass matrices with $\hat{M}_{N}^{ \pm}=\hat{M}_{N} \pm \frac{1}{2} \mu^{V}$ and $\mu^{V}=V_{R}^{T} \mu V_{R}^{T}$. The neutral mass eigenstates $\mathcal{N}^{\prime}=\left(\nu, N_{-}, N_{+}\right)^{T}$ correspond to three light neutrinos and three pairs of almost degenerate heavy neutrinos with mass eigenvalues $m_{N_{i}^{ \pm}}=\left(\hat{M}_{N}^{ \pm}\right)_{i i}=$ $\left(\hat{M}_{N}\right)_{i i} \pm \frac{1}{2}\left(\mu^{V}\right)_{i i}{ }^{2}$

Using Eqs (28), (29) the light neutrino mass matrix can be written as

$$
m_{\nu}=V_{L}^{T} M_{D}^{T} \frac{1}{M_{N}^{T}} \mu \frac{1}{M_{N}} M_{D} V_{L}
$$

Following the parametrization developed by Casas and Ibarra [18] we can now write $M_{D}$ as:

$$
M_{D}=M_{N} \frac{1}{\sqrt{\hat{\mu}}} \mathcal{R} \sqrt{m_{\nu}} V_{L}^{\dagger}
$$

Here the matrix $\mathcal{R}$ is an arbitrary complex orthogonal matrix. Rewriting $M_{N}$ using Eq. (28) one finds:

$$
V_{R}^{\dagger} M_{D}=\hat{M}_{N} U_{R}^{\dagger} \frac{1}{\sqrt{\hat{\mu}}} \mathcal{R} \sqrt{m_{\nu}} V_{L}^{\dagger},
$$

which express $V_{R}^{\dagger} M_{D}$ in terms of the low energy observables $m_{\nu}, V_{L}$ allowing us to reproduce the neutrino data. Notice that in practice, the arbitrariness of the matrix $\mathcal{R}$ is a consequence of the fact that for the nonminimal models, the Dirac mass matrix is arbitrary. This feature precludes a direct mapping of neutrino data onto $M_{D}$ in nonminimal models.

The mixing matrix $\mathcal{V}$ that relates the neutral mass eigenstates $\mathcal{N}^{\prime}$ and the interaction eigenstates $\mathcal{N}$ via $\mathcal{N}=$ $\mathcal{V} \mathcal{N}^{\prime}$ can be expressed in the seesaw approximation as [45]:

$$
\mathcal{V} \simeq\left(\begin{array}{ccc}
V_{L} & i \Theta_{L} & \Theta_{L} \\
0 & -\frac{i}{2} V_{R}^{*} & \frac{1}{2} V_{R}^{*} \\
-\sqrt{2} U_{R} \Theta_{L}^{\dagger} V_{L} & \frac{i}{\sqrt{2}} U_{R} & \frac{1}{\sqrt{2}} U_{R}
\end{array}\right)
$$

where $\Theta_{L}=\frac{1}{\sqrt{2}} M_{D}^{\dagger} V_{R} \hat{M}_{N}^{-13}{ }^{3}$

\footnotetext{
${ }^{2}$ Here the three pairs of - almost degenerate-neutrinos correspond to the so-called "quasi-Dirac" neutrinos $[24,45,46]$.

${ }^{3}$ The expressions of the couplings of the heavy neutrinos to the gauge bosons are given in [45].
} 


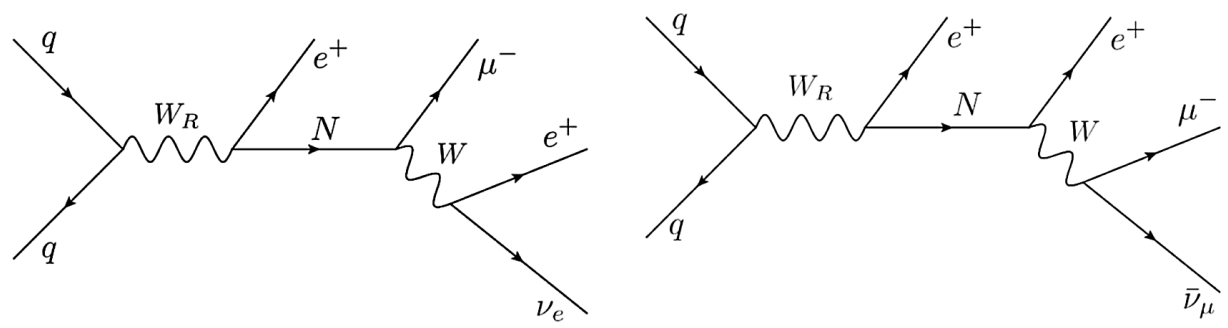

FIG. 1. Feynman diagrams for the $W_{R}$ production and leptonic decay $N \rightarrow e^{+} \mu^{-} \nu$, where $\nu$ can be either a $\nu_{e}$ or $\bar{\nu}_{\mu}$.

\section{COLLIDER SENSITIVITIES}

As discussed in the previous section, the most promising channel for the determination of the Dirac Yukawa coupling of neutrinos is the purely leptonic channel $p p \rightarrow$ $W_{R}^{ \pm} \rightarrow l^{ \pm} l^{ \pm} l^{\mp} \nu$. For purposes of illustration, we focus on the process $p p \rightarrow e^{+} N \rightarrow e^{+} \mu^{-} e^{+} \nu$ (see Fig. 1) rather than $p p \rightarrow \mu^{+} N \rightarrow \mu^{+} e^{-} e^{+} \nu$ in order to avoid the presence of an $e^{+} e^{-}$pair in the final state. The final state with different flavors for leptons of the same charge has a cleaner Standard Model background and also avoids events coming from the heavy neutrino decaying through the neutral currents (for example $p p \rightarrow W_{R}^{+} \rightarrow e^{+} N \rightarrow e^{+} \nu Z_{(R)}^{*} \rightarrow$ $\left.e^{+} e^{+} e^{-} \nu\right)$.

We study the main sources of background events (B) for the process $p p \rightarrow e^{+} N \rightarrow e^{+} \mu^{-} e^{+} \nu$ for different center of mass energies. In what follows, we discuss the LHC expected sensitivity to the branching ratio of HNs decaying into leptons at the LHC with $\sqrt{s}=13 \mathrm{TeV}$, the high energy LHC (HE-LHC) with $\sqrt{s}=28 \mathrm{TeV}$ and a $p p$ collider with $\sqrt{s}=100 \mathrm{TeV}$. We compare our cross section results with those obtained in Refs. $[15,16,47,48]$ for the $p p \rightarrow e^{+} N$ production and find agreement.

Assuming that the neutrinos in the final state cannot be distinguished, the decay width of heavy neutrinos into three leptons $\Gamma\left(N \rightarrow l^{ \pm} l^{\mp} \nu\right)$ is proportional to the heavy-light mixing and it is of the form

$$
\begin{aligned}
\Gamma\left(N \rightarrow l^{ \pm} l^{\prime \mp} \nu\right)= & \left(\left|\left(\Theta_{L}\right)_{l N}\right|^{2}+\left|\left(\Theta_{L}\right)_{l^{\prime} N}\right|^{2}\right) \frac{G_{F}^{2}}{96 \pi^{4} m_{N}} \\
& \times \int_{0}^{m_{N}^{2}} d x \frac{\pi\left(m_{N}^{2}-x\right)\left(m_{N}^{4}+x m_{N}^{2}-2 x^{2}\right)}{m_{N}^{2}\left(1-\frac{x}{M_{W}^{2}}\right)^{2}},
\end{aligned}
$$

where $m_{N}$ denotes the mass of the heavy neutrino. ${ }^{4}$ For illustration we assume $V_{L}=V_{R}^{*}$ and the indicative upper limit on light neutrino masses $\sum_{\nu} m_{\nu}=0.5 \mathrm{eV}$ [49]. In Fig. 2 we show the branching ratio of the heavy neutrino $N$ into $e^{+} \mu^{-} \nu$ as a function of the lightest heavy neutrino (HN) mass in the minimal left-right symmetric model. As can be

\footnotetext{
${ }^{4}$ In the inverse seesaw scenario, $m_{N}$ denotes collectively the pair of mass eigenvalues $m_{N^{ \pm}}$for $N=N_{ \pm}$.
}

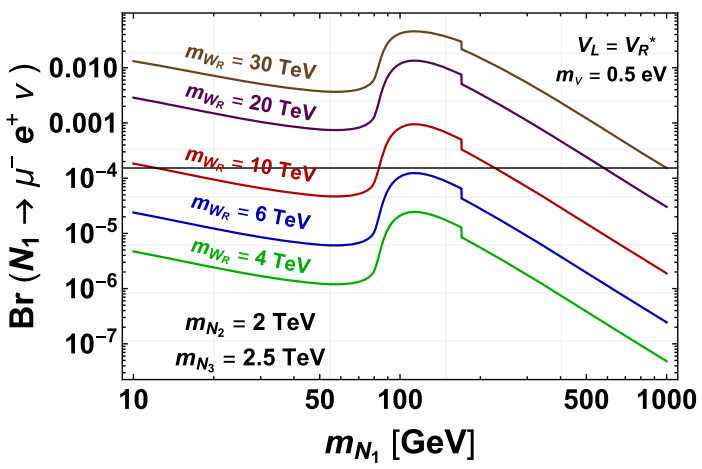

FIG. 2. Branching ratio of the purely leptonic decays of the heavy neutrino $N$ in the minimal left-right symmetric model. We use the indicative limit for light neutrino masses of $m_{\nu}=0.5 \mathrm{eV}$ [49].

seen from the figure, the branching ratio into leptons decreases as the heavy neutrino mass $m_{N}$ increases. This feature is due to the proportionality of the leptonic branching ratio to $\Theta_{L}^{2}$ [see Eq. (35)], which in turn is proportional to $1 / m_{N}$ - see Eq. (26).

Another important feature is the increase of the leptonic branching ratio as the $W_{R}$ boson mass increases. This occurs because the dominant process with one lepton and two jets has an additional suppression of $M_{W_{R}}$. The net effect is to make the branching ratio into leptons increase when the $W_{R}$ boson mass increases. Finally, the bump when $m_{N} \sim M_{W}$ is due to the transition from three body decay to a two-body decay through an on-shell $W$ boson. The drop in the decay rate due to the top quark threshold is also evident.

Regarding the processes shown in Fig. 1, we find two issues that may affect the selection efficiency ${ }^{5}$ of the signal: (1) the two origins of the $\mu^{-}$, which is an interpretation issue and (2) the possible jet fake background:

(1) The origin of the $\mu^{-}$: there are two possibilities

$$
p p \rightarrow e^{+} N \rightarrow e^{+} \mu^{-}\left(W^{+} \rightarrow e^{+} \nu_{e}\right),
$$

and

\footnotetext{
${ }^{5}$ We define the efficiency as the number of events kept after a given cut, divided by the initial number of events without cuts.
} 


$$
p p \rightarrow e^{+} N \rightarrow e^{+} e^{+}\left(W^{-} \rightarrow \mu^{-} \bar{\nu}_{\mu}\right) .
$$

Namely, the final state muon can be directly produced in the decay of the heavy neutrino $N$ or it can also be produced in the decay of the $W$ boson that comes from the decay of the heavy neutrino $N$. Notice that the lepton flavor cannot be used to discriminate among the two processes since the light neutrino goes undetected.

For $m_{N}>m_{W}$, the transverse mass of the subleading positron and missing transverse energy $E_{T}$ system $m_{T}\left(e_{\text {sub }}^{+} E_{T}\right)$ may be helpful for discriminating between the two processes. In the process in Eq. (36) the subleading positron comes primarily from the decay of an on-shell $W$ boson, so the transverse mass of the subleading positron and missing transverse energy system will have a sharp decline around the mass of the $W$ boson. On the contrary, in the process in Eq. (37), the subleading positron directly comes from the decay of heavy neutrino $N$, so one may expect a broader distribution of this transverse mass. In principle, then, implementing a cut on this transverse mass near $m_{W}$ should remove a significant portion of events from the process in Eq. (37) while retaining most events coming from the process in Eq. (36). We expect this method will be useful for $m_{N} \gg m_{W}$. How can one distinguish between these two channels when $m_{N}$ is near $m_{W}$ ? One may in principle perform an additional analysis replacing the cut $m_{T}\left(e_{\text {sub }}^{+} E_{T}\right) \rightarrow$ $m_{T}\left(\mu^{-} E_{T}\right)$. Since the efficiencies for each individual channel in Fig. 1 are different in both analysis, one can in principle extract the individual matrix elements for each individual channel. In any case, the main point we want to convey is that the trilepton signal is completely predicted in the mLRSM in terms of the heavy and light neutrino mass matrices irrespectively on whether this distinction can be done. Hence, in what follows we will not distinguish the two leptonic channel in Fig. 1.

For $m_{N}<m_{W}$ the decay goes through an offshell $W$ boson. In this case, it seems at first glance more difficult to distinguish between these two contributions. In principle, one cannot determine the origin of the muon for a single event. However, a way to determine the proportion of $\mu^{-}$from each channel by measuring a particular forwardbackward asymmetry with an ensemble of events has been proposed in Ref. [50]. In this work, we follow a different approach. In both cases mentioned above, when deriving the sensitivity to the decay branching ratio $\operatorname{Br}\left(N \rightarrow e^{+} \mu^{-} \nu\right)$, we first estimate the efficiency for each channel shown in Fig. 1 (see Fig. 4) and subsequently compute the average efficiency by weighting each channel with the corresponding probability of occurrence.

(2) Since the mixing parameter $\Theta_{L}$ may be quite small, there may be a non-negligible jet-fake background coming from the process $p p \rightarrow W_{R}^{ \pm} \rightarrow l^{ \pm} l^{ \pm} j j$, where one of the jets is misidentified as either an electron or a muon, since we did not reject extra jets in our analysis. Notice that the branching ratio for this channel is by far the dominant one. Therefore, it can mimic the purely leptonic signal with one of the jets faking to leptons, which may be a contamination and decrease the sensitivity to the heavy-light mixing. As discussed below, we address this issue by implementing cuts on the missing transverse energy $E_{T}$ and the transverse mass of the subleading electron and $E_{T}$ system. We find that for heavy neutrino masses $m_{N}<1 \mathrm{TeV}$, this channel is subdominant with respect to the trilepton channel.

For the signal generation, we use the extension of the FEYNRULES package [51] for the minimal LR model used in Ref. [52] and expanded in Ref. [53]. The signal and background events were generated at LO using MADGRAPH 5 [54], Pythia 6 [55] for hadronization, and DelPhes 3 [56] for detector simulation, using the JetFake module developed in [53]. The dominant sources of background are found to be $t \bar{t} W, t \bar{t}(j)$ (with a jet faking a lepton) and $W W W(j)$, while $W Z(j), t \bar{t} Z$ and $Z / \gamma(j)$ (with charge flip and a jet faking a muon) are subdominant. The $j$ in the parenthesis means that we generated the corresponding background with one matched jet. Tables II, III and IV show the cut flow (see below) for the main sources of background for this process, together with two signal

TABLE I. Selection criteria used to reduced the SM background for $100 \mathrm{TeV}$. For $13 \mathrm{TeV}$ and $28 \mathrm{TeV}$ we apply the same cuts, excepting that $P_{T, e^{+}}^{\text {lead }}>100 \mathrm{GeV}$.

\begin{tabular}{ll}
\hline \hline Cut description & \\
\hline$e^{+} e^{+} \mu^{-}$, no $b$ jets and no additional leptons & Signal selection \\
$p_{T, e^{+}}^{\text {lead }}>200 \mathrm{GeV}, p_{T, e^{+}}^{\text {sub }}>100 \mathrm{GeV}, p_{T, \mu^{-}}^{\text {lead }}>100 \mathrm{GeV}$ & Reduces all backgrounds \\
$\mathscr{E}_{T}>100 \mathrm{GeV}$ & Reduces mostly $t \bar{t}(j)$ and $Z / \gamma(j)$ \\
$\left.\left|m_{\text {inv }}\left(e^{+} e^{+}\right)-91.2\right|\right)>10 \mathrm{GeV}$ & Reduces mostly $W Z(j)$ \\
$m_{T}\left(e_{\text {sub }}^{+} E_{T}\right)<150 \mathrm{GeV}$ & Enhances channel shown in Fig. 1 (left) \\
$m_{T}\left(e^{+} e^{+} \mu^{-} E_{T}\right)>M_{W_{R}} / 2$ & Reduces all backgrounds \\
\hline \hline
\end{tabular}




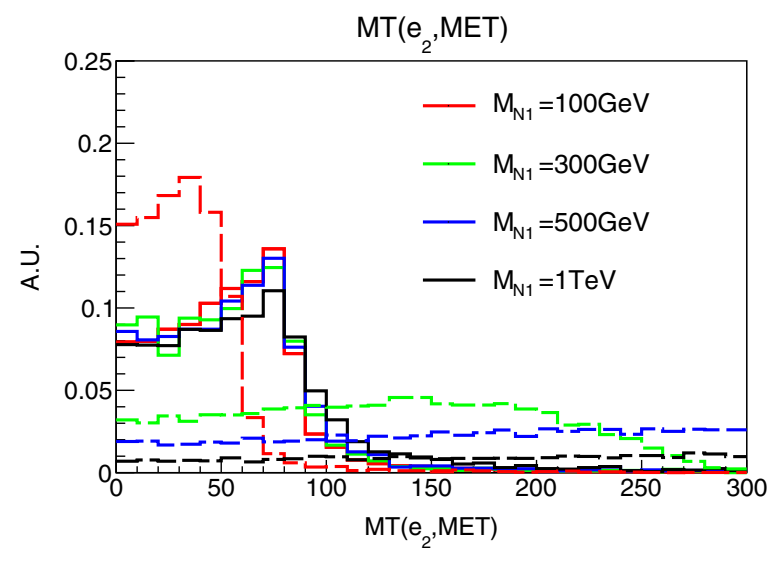

FIG. 3. The distribution of the transverse mass of the subleading positron and missing $E_{T}$ at $100 \mathrm{TeV}$ pp-collider with benchmark point $M_{W_{R}}=6 \mathrm{TeV}, \quad m_{N_{2}}=2 \mathrm{TeV}, \quad m_{N_{3}}=$ $2.5 \mathrm{TeV}$. Different colors represents different masses of $m_{N_{1}}$, the solid and dashed curves represent the events coming from the left and right diagram in Fig. 1 respectively.

benchmark points, for $13 \mathrm{TeV}, 28 \mathrm{TeV}$ and $100 \mathrm{TeV}$, respectively. As already remarked, we assume the left and right leptonic mixing matrix to satisfy $V_{L}=V_{R}^{*}$. Some of the backgrounds for this process were studied in Ref. [22]. In our analysis, further sources of backgrounds are included mostly due to the charge misidentification probability that becomes more important at higher $p_{T}$. We compare our $W Z$ and triple boson $(W W W)$ backgrounds with the CMS estimates from Ref. [22]. In particular, we compare with the second last bin in the left panel of Figure A.3 from Ref. [22], which turns out to be closer to the kinematic region in our analysis, and find an agreement for $W Z$ and about half of the yield for the $W W W$ background. This difference is consistent with the $50 \%$ uncertainty quoted for the estimate for triboson production.

A description of the selection criteria is shown in Table I. We first demand that each event contains exactly two positrons, one muon, and no b-tagged jets. Events with

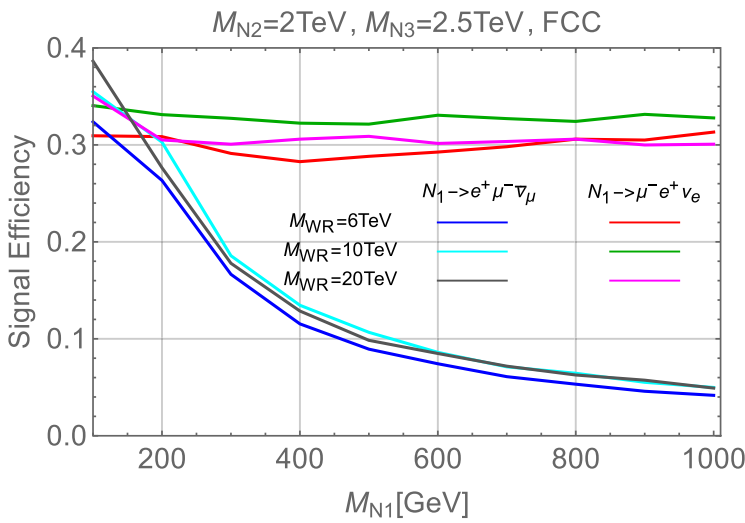

extra jets that are not b-tagged are retained. Second, we select events with high transverse momentum $p_{T}$ for the leptons and large missing transverse energy $E_{T}$ in order to reduce many of the backgrounds. Then we require the reconstructed invariant mass of the positron pair $m_{\text {inv }}\left(e^{+} e^{+}\right)$to be outside the $\mathrm{Z}$ boson mass peak, reducing the background coming from $Z \rightarrow e^{+} e^{-}$when the electron charge is misidentified.

The next cut in Table I is on the transverse mass of the positron and missing energy $m_{T}\left(e_{\text {sub }}^{+} E_{T}\right)$. We enforce the reconstructed transverse mass of the subleading positron and missing energy to be less than $150 \mathrm{GeV}$. In principle, if the momentum of the leptons are exactly reconstructed, then $m_{T}\left(e_{\text {sub }}^{+} E_{T}\right)$ will not exceed the mass of the $W$ boson. However, due to smearing effects, the distribution of this transverse mass is broadened. This is why we choose the cut on this variable to be larger than the $W$ boson mass. In Fig. 3 we show the $m_{T}\left(e_{\text {sub }}^{+} E_{T}\right)$ distribution for different masses of the heavy neutrino $N_{1}$ and $M_{W_{R}}=6 \mathrm{TeV}$ for a $100 \mathrm{TeV}$ pp-collider. One can observe from these distributions that imposing a cut on this transverse mass can effectively discriminate the two diagrams in Fig. 1 if the mass of the $N_{1}$ is sufficiently large. As noted earlier, this discrimination can be achieved because the positron from the heavy neutrino decay comes mostly from an on-shell $W$ boson decay in the process in Eq. (37). The signal efficiencies are different for the two processes in Fig. 1. In Fig. 4 we show the signal efficiency for each channel individually as well as the averaged efficiencies with different relative strength of the two channels characterized by the parameter $r$ defined below:

$$
r \equiv \frac{\operatorname{Br}\left(N_{1} \rightarrow e^{+}\left(W^{-} \rightarrow \mu^{-} \bar{\nu}_{\mu}\right)\right)}{\operatorname{Br}\left(N_{1} \rightarrow \mu^{-}\left(W^{+} \rightarrow e^{+} \nu_{e}\right)\right)} .
$$

As one can see from the left plot in Fig. 4, the efficiency of the channel shown in Eq. (37) decreases as the mass of the

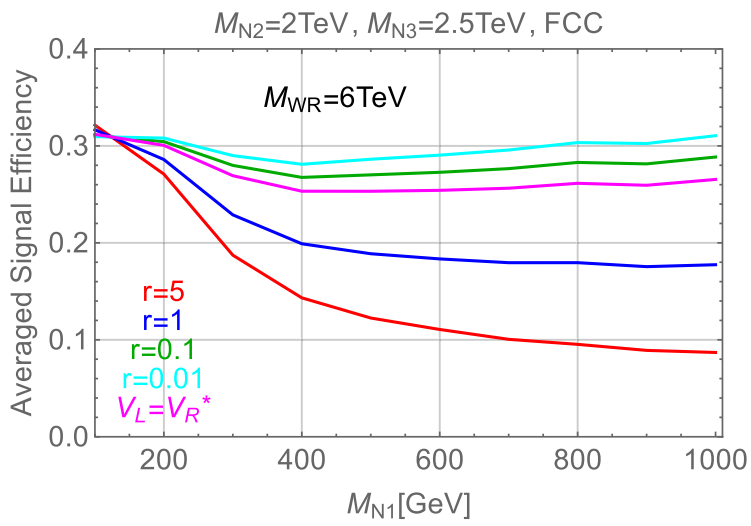

FIG. 4. The signal efficiencies for the benchmark point: $m_{N_{2}}=2 \mathrm{TeV}, m_{N_{3}}=2.5 \mathrm{TeV}$. The left plot shows the signal efficiencies for each channel with different mass of $W_{R}$ and $N_{1}$. The right plot shows the signal efficiencies for different relative strengths $r$ of the two channels (defined in Eq. (38), with $M_{W_{R}}=6 \mathrm{TeV}$. 


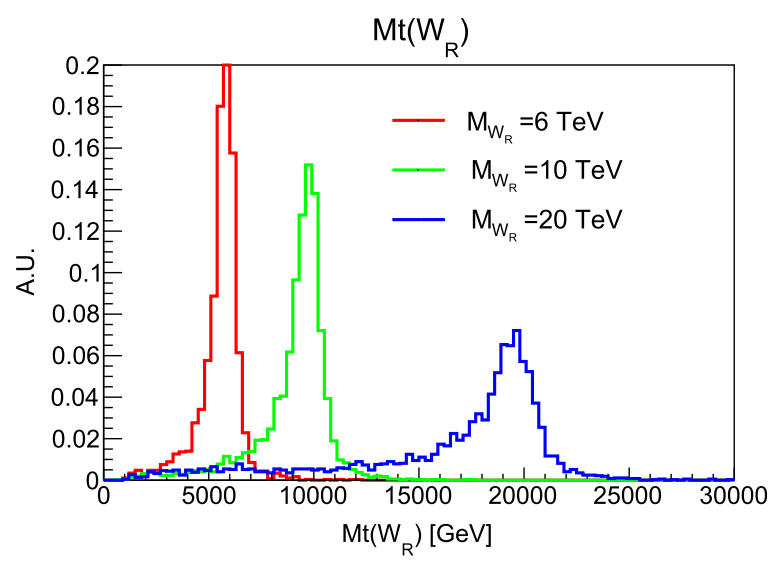

FIG. 5. The distribution of the reconstructed transverse mass of $W_{R}$ at a $100 \mathrm{TeV}$ pp-collider, with benchmark point $m_{N}=$ $300 \mathrm{GeV}, m_{N_{2}}=2 \mathrm{TeV}, m_{N_{3}}=2.5 \mathrm{TeV}$. Different colors represents different masses of $W_{R}$.

$N_{1}$ increases. This is mainly due to the cut $m_{T}\left(e_{\text {sub }}^{+} E_{T}\right)<$ $150 \mathrm{GeV}$ shown in Table I, which helps to discriminate between the two channels shown in Eqs. (36) and (37).

The last selection in Table I is a cut on the transverse mass of the $e^{+} e^{+} \mu^{-} E_{T}$ system, since for an on-shell $W_{R}$ boson, the transverse mass distribution is peaked at $M_{W_{R}}$ (see Fig. 5), where the SM backgrounds give a negligible contribution. The rejection of the backgrounds was effectively achieved by using the cut $m_{T}\left(e^{+} e^{+} \mu^{-} E_{T}\right)>M_{W_{R}} / 2$ shown in Table I. In this way, most of the signal events are kept while a significant portion of the backgrounds is rejected. Furthermore, this cut also guarantees that the SM backgrounds become even more suppressed when searching for a $W_{R}$ boson with higher mass.

For the charge flip probability, we take the current ATLAS performance in Ref. [57], which parameterizes the flip probability $P$ as the product of functions of $\eta$ and $P_{T}: P=f(\eta) \times \sigma\left(P_{T}\right)$. Also we assume that $\sigma\left(P_{T}\right)$ for $P_{T}>400 \mathrm{GeV}$ keeps the same value as that in the bin $(200,400) \mathrm{GeV}$. For our analysis of the $100 \mathrm{TeV}$ collider reach, we use the same charge flip probability, as we are not aware of a more realistic estimation having appeared in the literature to date.

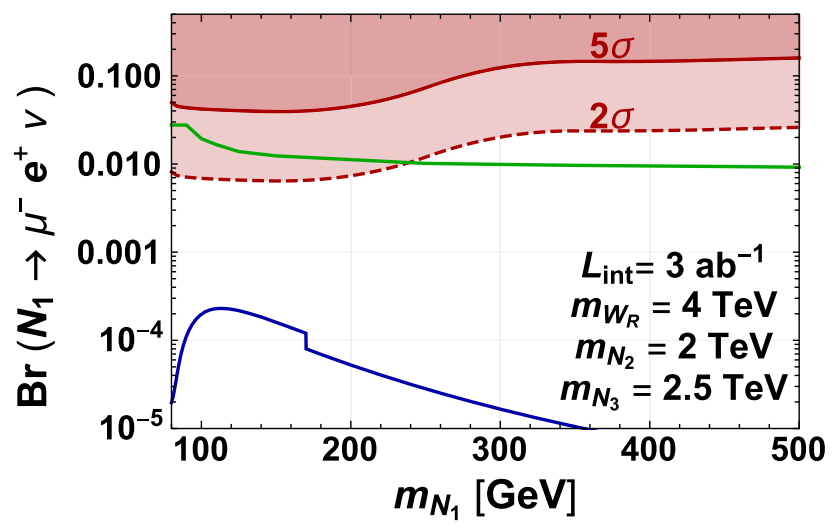

FIG. 7. LHC reach to the branching ratio of the purely leptonic decays of the heavy neutrino. The blue (green) line denotes the branching ratio within the minimal (nonminimal) LR model and the shadowed thick(dashed) regions show the reach at $5 \sigma(2 \sigma)$, for an integrated luminosity of $L_{\text {int }}=3 \mathrm{ab}^{-1}$ and center of mass energy $\sqrt{s}=13 \mathrm{TeV}$. We assume $V_{L}=V_{R}^{*}$ and the upper limit on light neutrino masses of $m_{\nu}=0.5 \mathrm{eV}$ [49]. For the nonminimal model we have set $\mathcal{R}=U_{R}=I$ and $\mu=10^{-4} \mathrm{GeV}$. Exclusion contours are obtained by using the condition $S / \sqrt{S+B}=2,5$ respectively.

A comment on a possible future refinement that can be made in the analysis and that exploits a particular kinematic feature of the signal. For HN masses much smaller than the $W_{R}$ boson mass, the decay products coming from the $\mathrm{HN}$ are merged and form what it has been coined as a lepton jet [21]. In Fig. 6, we show $\Delta R \equiv \sqrt{\Delta \phi^{2}+\Delta \eta^{2}}$ for the decay products of the process shown in Fig. 1. Since $\Delta R\left(\mu e_{2}\right)$ for the signal is peaked at smaller values (around $\Delta R \sim 5 \times$ $10^{-2}$ for $m_{N}=100 \mathrm{GeV}$ and for $\left.M_{W_{R}}=6,10,20 \mathrm{TeV}\right)$ than $\Delta R\left(\mu e_{1}\right)$ and $\Delta R\left(e_{1} e_{2}\right)$, we see that the positron with smaller energy is mainly coming from the $\mathrm{HN}$ decay. This kinematic variable has been previously proposed in Ref. [32]. Although we did not need to use this kinematic feature in our analysis, it is worth to keep it in mind, since it is one of the more distinctive topological features of the signal in the mass range considered in this work and it may be used to reject further backgrounds.
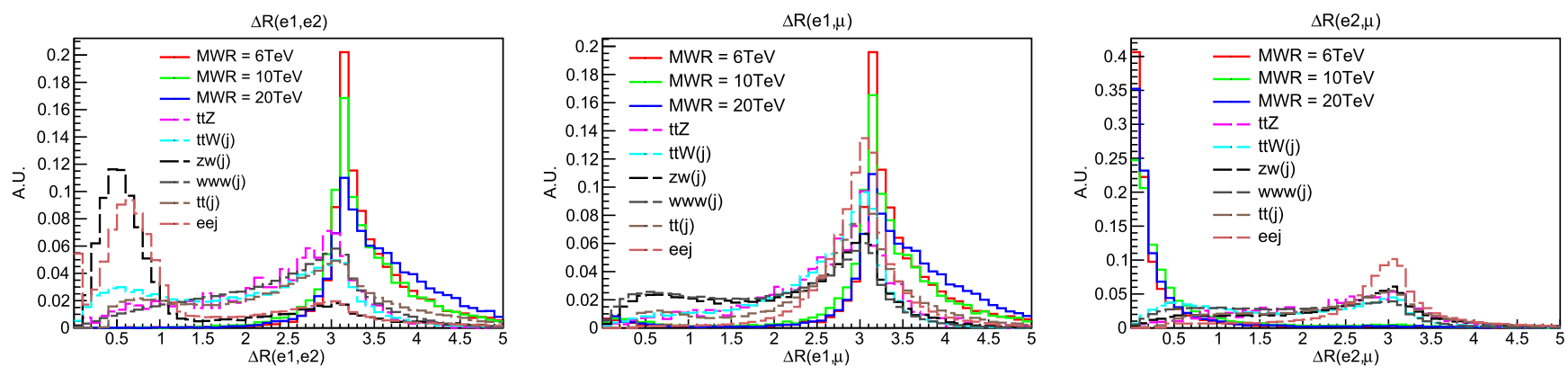

FIG. 6. $\Delta R$ for the decay products of the process shown in Fig. 1. $e_{1}$ and $e_{2}$ refers to the harder and softer positrons sorted by transverse momentum. We assume $m_{N}=100 \mathrm{GeV}$ and plot the signal for different values of $M_{W_{R}}$. 
TABLE II. SM background processes at $13 \mathrm{TeV}$ and $3 \mathrm{ab}^{-1}$ for the trilepton signal $e^{+} e^{+} \mu^{-} \nu$ and $M_{W_{R}}=4 \mathrm{TeV}$, for two benchmark values of the heavy neutrino masses assuming $\left(\Theta_{L}\right)_{\mu N}=(\Theta)_{e N}=\frac{1}{\sqrt{2}}$. Backgrounds ending with $(j)$ were simulated with one matched jet. The charge misidentification probability has been taken from current ATLAS result from Ref. [57].

\begin{tabular}{|c|c|c|c|c|c|c|c|c|}
\hline \multirow[b]{2}{*}{$\sqrt{s}=13 \mathrm{TeV}$} & \multicolumn{6}{|c|}{ Backgrounds } & \multicolumn{2}{|c|}{ Signal } \\
\hline & $t \bar{t} Z$ & $\bar{t} \bar{t} W$ & $t \bar{t}(j)$ & $W Z(j)$ & $3 W$ & $Z / \gamma(j)$ & $m_{N}(100 \mathrm{GeV})$ & $m_{N}(500 \mathrm{GeV})$ \\
\hline$e^{+} e^{+} \mu^{-}$(b-veto) & 11.8 & 74.9 & 23058 & 24.8 & 6.71 & 901 & 1293 & 371 \\
\hline$P_{T}$ cuts & 0.325 & 3.75 & 216 & 0.215 & 2.33 & 5.31 & 825 & 253 \\
\hline $\mathscr{E}_{T} \mathrm{GeV}$ & 0.158 & 1.85 & 117 & 0.0761 & 1.06 & 0.0911 & 646 & 188 \\
\hline$m_{\mathrm{inv}}\left(e^{+} e^{+}\right)$ & 0.155 & 1.82 & 113 & 0.0761 & 1.05 & 0 & 646 & 188 \\
\hline$m_{T}\left(e_{\text {sub }}^{+} E_{T}\right)$ & 0.0582 & 0.743 & 48.4 & 0.0277 & 0.491 & 0 & 622 & 176 \\
\hline$m_{T}\left(e^{+} e^{+} \mu^{-} \mathbb{E}_{T}\right)$ & 0 & $7.82 \times 10^{-3}$ & 0 & 0 & 0.0169 & 0 & 597 & 158 \\
\hline
\end{tabular}

In Fig. 6, one can see that since $\Delta R\left(e_{2} \mu\right)<0.5$, it falls into the definition of a muon jet given in Ref. [21]. This is in fact the same requirement of Ref. [38] and the main discriminating features with respect to those references are: (i) we focus on the kinematic regions where the $W_{R}$ boson is produced nearly on-shell. In this case, due to the heaviness of the $W_{R}$ boson, the prompt lepton from the decay still gets much higher $p_{T}$ than that decay from the ordinary $\mathrm{W}$ boson. This is why we set a much higher cut on the lepton $p_{T}$ than in Refs. [21,38] (see Table I). Especially for the future collider experiments, the resonance peak of the $W_{R}$ can be seen clearly, which would be a definite difference compare with the model without the $W_{R}$ boson. (ii) At the $14 \mathrm{TeV}$ LHC, most of the events are coming from the off-shell decay where the resonance peak cannot be clearly identified. The high value of the transverse momentum cut on the lepton in Table I is still useful to suppress the events coming from the ordinary $W$ boson decay, though we are not be able to confirm the existence of the $W_{R}$ if there is an excess observed in the experiment. (iii) The typical mass of the lightest heavy neutrino is $\mathrm{O}(100) \mathrm{GeV}$ in our study unlike Refs. [21,38] with $\mathcal{O}(10) \mathrm{GeV} \mathrm{HN}$ masses. This means that the signal in Refs. [21,38] is usually displaced, unlike our signal, which is prompt.

\section{A. Sensitivity at the LHC}

The LHC sensitivity to the branching ratio into the purely leptonic channel is shown in Fig. 7, with the corresponding cut flow for representative two signal points given in Table II. ${ }^{6}$

In our calculations we assumed $V_{L}=V_{R}^{*}$ and set $\mathcal{R}=U_{R}=I, \mu=10^{-4} \mathrm{GeV}$ for the nonminimal model. This choice of parameters corresponds to $r \sim 1$ in both

\footnotetext{
${ }^{6}$ In this analysis, we studied the sensitivity of the trilepton signal for masses $m_{N} \gtrsim 80 \mathrm{GeV}$. A similar analysis can be carried out for smaller masses, although in this case the $N$ could decay with a displaced vertex, as previously studied in Refs. [48,58-61].
}

scenarios. ${ }^{7}$ As expected the maximum reach is obtained for $M_{W_{R}}=4 \mathrm{TeV}$, for a wide range of the heavy neutrino mass, with the branching ratio reach extending down to $\sim 10^{-2}$ at $2 \sigma$ significance.

The solid blue and green curves show the expected branching ratios in the mLRSM and nonminimal models, respectively. It is clear that for the mLRSM, the branching ratio lies well below the sensitivity the HL-LHC $\left(L_{\text {int }}=\right.$ $\left.3 \mathrm{ab}^{-1}\right)$. This suppression with respect to the LHC reach follows from the small values of $M_{D} / M_{N}$ needed for consistency with the observed small scale of the light neutrino masses that imply, in turn, small values of the heavy-light neutrino mixing angle. This suppression is not necessarily true in an inverse seesaw scenario. In the latter case, the smallness of the neutrino masses can be attributed to small values of the $\mu$ parameter [see Eq. (31)] without requiring the exceedingly small values of $M_{D} / M_{N}$ [24,45,62]. This feature can be seen explicitly in Fig. 7, where we have obtained the green curve by choosing $\mu=10^{-4} \mathrm{GeV}$. Therefore, we can conclude that a signal of the purely leptonic channel at the LHC would be an indication of a nonminimal realization of the LR symmetry.

\section{B. Sensitivity at the HE-LHC}

We now consider the reach of the proposed energy upgrade of the LHC to $28 \mathrm{TeV}$ center of mass energy (HELHC). In Fig. 8 we show the expected reach at the HE-LHC for two values of the $W_{R}$ boson mass. We see from the figure that heavy light mixing can be excluded at $2 \sigma$ for heavy-light mixing of the order of $10^{-3}$ for $M_{W_{R}}=6 \mathrm{TeV}$ and of the order of $10^{-2}$ for $M_{W_{R}}=10 \mathrm{TeV}$. As in the case of the high luminosity LHC (HL-LHC), the minimal LR

\footnotetext{
${ }^{7}$ In the inverse seesaw scenario different values of $r \sim[0,1]$ are possible even if we assume $V_{L}=V_{R}^{*}$. This is because the lepton number violating process $p p \rightarrow W_{R}^{+} \rightarrow e^{+} e^{+} \mu^{-} \bar{\nu}_{\mu}$ in Eq. (37) might be suppressed with respect to the lepton number conserving process $p p \rightarrow W_{R}^{+} \rightarrow e^{+} \mu^{-} e^{+} \nu_{e}$ in Eq. (36) for $\mu<\Gamma_{N}$, being $\Gamma_{N}$ the decay width of the heavy neutrino (see the discussion in Refs. [45,62]).
} 

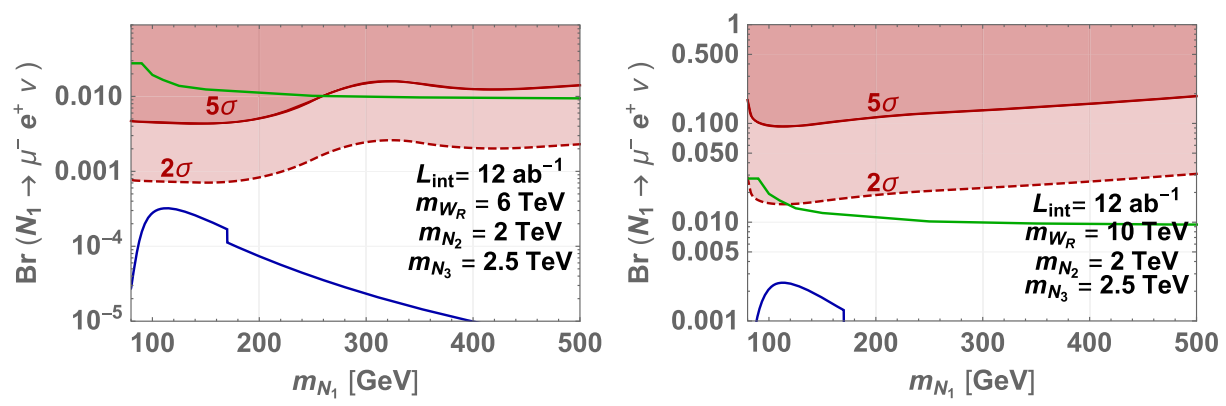

FIG. 8. HE-LHC reach to the branching ratio of the purely leptonic decays of the heavy neutrino. The blue (green) line denotes the branching ratio within the minimal (nonminimal) LR model and the shadowed thick(dashed) regions show the reach at $5 \sigma(2 \sigma)$, for an integrated luminosity of $L_{\text {int }}=12 \mathrm{ab}^{-1}$ and center of mass energy $\sqrt{s}=28 \mathrm{TeV}$. We assume $V_{L}=V_{R}^{*}$ and the upper limit on light neutrino masses of $m_{\nu}=0.5 \mathrm{eV}$ [49]. For the nonminimal model we have set $\mathcal{R}=U_{R}=I$ and $\mu=10^{-4} \mathrm{GeV}$.

scenario is not expected to give any observable signal in the purely leptonic channel, so a positive signal would point to a nonminimal realization of the LR model. Finally and for the purpose of illustration, in Table III we show the cut flow for the main background processes and two benchmark points for the signal.

\section{Sensitivity at the $100 \mathrm{TeV} p p$ collider}

We now turn to the reach of a $100 \mathrm{TeV} p p$ collider, as shown in Fig. 9. The corresponding cut flow is given in Table IV.

In contrast to the situations for the HL-LHC and HELHC, a $100 \mathrm{TeV} p p$ collider could observe the trilepton channel for a sufficiently light $W_{R}$. As one increases the $W_{R}$ mass, the range of $\mathrm{HN}$ masses for which the trilepton channel is accessible decreases. From the left panel of Fig. 9, we see that for $M_{W_{R}}=6 \mathrm{TeV}$ the purely leptonic signal can be discovered(excluded) with $5 \sigma(2 \sigma)$ sensitivity for heavy neutrino masses below $300 \mathrm{GeV}(450 \mathrm{GeV})$. It is, thus, possible that one might discover the $W_{R}$ boson at the LHC (with $M_{W_{R}}<6 \mathrm{TeV}$ ) using the two lepton and two jets channel [48] yet require a $100 \mathrm{TeV} p p$ collider to probe the $\mathrm{HN}$ mass generation by measuring the trilepton signal. For heavier $W_{R}$ masses, both discovery of the RH $W$ boson and testing the mLRSM Yukawa sector would require a next generation collider. For $M_{W_{R}}=10 \mathrm{TeV}$, for example, the purely leptonic signal can be discovered(excluded) with $5 \sigma(2 \sigma)$ sensitivity for heavy neutrino masses below $260 \mathrm{GeV}(400 \mathrm{GeV})$. Finally, for $M_{W_{R}}=20 \mathrm{TeV}$ heavy neutrino masses up to $200 \mathrm{GeV}$ can be excluded at $2 \sigma$.

Notice that from this analysis it emerges that the region of phenomenological interest is for $m_{N} \ll M_{W_{R}}$, although both $\mathrm{HN}$ and $W_{R}$ are at the large $v_{R}$ scale. This situation presents no problems. Indeed, HN masses are arbitrary, apart from direct experimental limits. From a theoretical perspective, because of chiral symmetry, fermion masses are technically natural, so one may choose them to be arbitrarily light. Furthermore, there is no need of any finetuning even between the type I and type II contribution to light neutrino masses in order to have relatively light heavy neutrinos, as discussed in Ref. [40].

It is true that scale ratios may be invoked in order to explain why a coupling is small, and this of the case in the GUT embedding of the mLRSM. That being said, in any case, TeV scale LR symmetry is perfectly well motivated theoretically and phenomenologically, as argued in Ref. [40]. In fact, in Ref [64] very light neutrinos with $m_{N} \sim \mathrm{keV}$ and $M_{W_{R}} \sim \mathrm{TeV}$, has been previously considered as the candidates of warm dark matter of the universe. Nevertheless, it now remains up to the results of experiment

TABLE III. SM background processes at $28 \mathrm{TeV}$ and $12 \mathrm{ab}^{-1}$ for the trilepton signal $e^{+} e^{+} \mu^{-} \nu$ and $M_{W_{R}}=6 \mathrm{TeV}$, for two benchmark values of the heavy neutrino masses assuming $\left(\Theta_{L}\right)_{\mu N}=(\Theta)_{e N}=\frac{1}{\sqrt{2}}$. Backgrounds ending with $(j)$ were simulated with one matched jet. The charge misidentification probability has been taken from current ATLAS result from Ref. [57].

\begin{tabular}{|c|c|c|c|c|c|c|c|c|}
\hline \multirow[b]{2}{*}{$\sqrt{s}=28 \mathrm{TeV}$} & \multicolumn{6}{|c|}{ Backgrounds } & \multicolumn{2}{|c|}{ Signal } \\
\hline & $t \bar{t} Z$ & $t \bar{t} W$ & $t \bar{t}(j)$ & $W Z(j)$ & $3 W$ & $Z / \gamma(j)$ & $m_{N}(100 \mathrm{GeV})$ & $m_{N}(500 \mathrm{GeV})$ \\
\hline$e^{+} e^{+} \mu^{-}$(b-veto) & 286 & 882 & 615657 & 440 & 56.6 & 3139 & 8766 & 3208 \\
\hline$P_{T}$ cuts & 9.93 & 60.0 & 8791 & 6.3 & 22.9 & 37.9 & 7000 & 2474 \\
\hline $\mathbb{E}_{T} \mathrm{GeV}$ & 5.18 & 34.1 & 5115 & 2.29 & 12.2 & 2.33 & 6037 & 2092 \\
\hline$m_{\text {inv }}\left(e^{+} e^{+}\right)$ & 5.00 & 33.6 & 4986 & 2.29 & 12.2 & 0.608 & 6037 & 2092 \\
\hline$m_{T}\left(e_{\mathrm{sub}}^{+} \boldsymbol{E}_{T}\right)$ & 2.14 & 13.7 & 2297 & 0.497 & 5.83 & 0 & 5678 & 1883 \\
\hline$m_{T}\left(e^{+} e^{+} \mu^{-} \mathbb{E}_{T}\right)$ & 0 & 0.028 & 3.00 & 0 & 0.13 & 0 & 5555 & 1800 \\
\hline
\end{tabular}



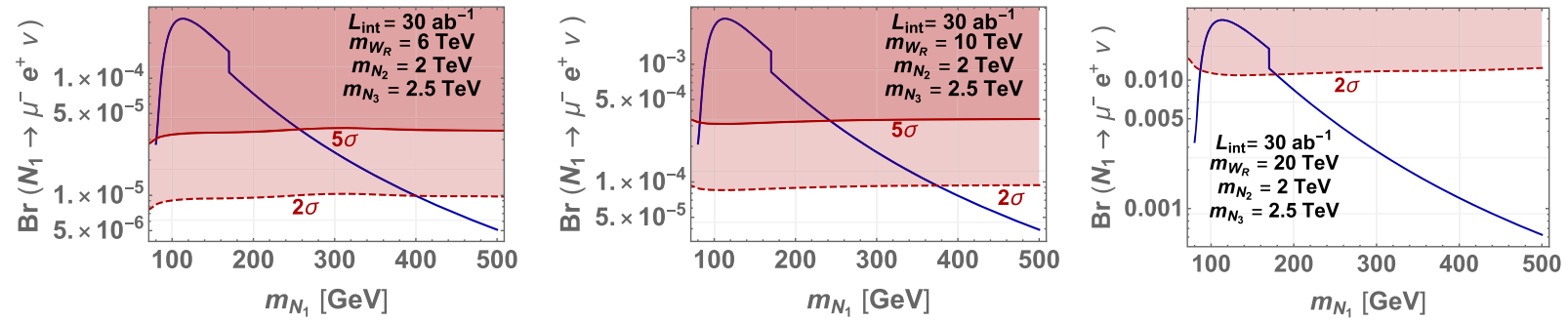

FIG. 9. $100 \mathrm{TeV} p p$ collider reach to the branching ratio of the purely leptonic decays of the heavy neutrino. The blue line denotes the branching ratio within the mLRSM and the shadowed thick(dashed) regions show the reach at $5 \sigma(2 \sigma)$, for an integrated luminosity of $L_{\text {int }}=30 \mathrm{ab}^{-1}$ and center of mass energy $\sqrt{s}=100 \mathrm{TeV}$. We assume $V_{L}=V_{R}^{*}$ and the upper limit on light neutrino masses of $m_{\nu}=0.5 \mathrm{eV}[49]$.

to determine the values of the $W_{R}$ and HN masses if leftright symmetry is realized in nature.

A comment about our benchmark is in order: a full analysis, as the one performed by ATLAS in Ref. [65] would look the cases when the HN are lighter and heavier than the $W_{R}$ boson. When $m_{N}<M_{W_{R}}$ the invariant mass of the $W_{R}$ boson can be reconstructed using the invariant mass of the $e e \mu E_{T}$ system. This is the case of interest for this work. When $m_{N}>M_{W_{R}}$, the cross section for the trilepton channel is kinematically suppressed with respect to the onshell $W_{R}$ boson case, since there is no enhancement from the $W_{R}$ boson peak.

\section{CONNECTION BETWEEN THE DIRAC MASS, THE HEAVY AND LIGHT NEUTRINO MASSES AND LOW ENERGY EXPERIMENTS}

In this section, we discuss what the expected sensitivity obtained in the previous section implies for the Dirac mass term of the minimal model. Summarizing, we have the two following important points regarding the connection between the Dirac mass and the heavy and light neutrino masses:

(1) The trilepton search can determine the mixing between the heavy and light neutrinos $\Theta_{L}$ in Eqs. (21) and (22) which, then, can be related to the Dirac mass $M_{D}$ using Eq. (15) once $M_{N}$ is known. The same conclusions apply for the nonminimal setup, where the heavy light mixing is of the form $\Theta_{L}=\frac{1}{\sqrt{2}} M_{D}^{\dagger} V_{R} \hat{M}_{N}^{-1}$ - see Eq. (34).

(2) The connection between $\Theta_{L}, M_{D}, M_{N}$ and $M_{\nu}$ is direct in the minimal model, as can be seen, for instance, from Eq. (26) for $\mathcal{C}$ as the LR symmetry. In the nonminimal model, relating experimentally accessible neutrino mass parameters with the Lagrangian mass parameters is far less direct due to the arbitrary, complex, orthogonal matrix $\mathcal{R}$ shown in Eq. (33).

Therefore and for the mLRSM, it is interesting to compare the exclusion lines obtained in Fig. 9 with the prediction one can make in this case. In Fig. 10 (left panel) we plot the combination $\left|\left(\Theta_{L}\right)_{N \mu}\right|^{2}+\left|\left(\Theta_{L}\right)_{N e}\right|^{2}$ given by Eq. (15), as a function of $m_{N}$ together with the $2 \sigma$ and $5 \sigma$ significance regions expected at the $100 \mathrm{TeV} p p$ collider. We see that at the $100 \mathrm{TeV} p p$ collider, the heavy-light mixing $\Theta$ can be probed for values as small as $\left|\left(\Theta_{L}\right)_{N \mu}\right|^{2}+\left|\left(\Theta_{L}\right)_{N e}\right|^{2} \sim 10^{-12}$. Notice that since $\left|\left(\Theta_{L}\right)_{N \mu}\right|^{2}+\left|\left(\Theta_{L}\right)_{N e}\right|^{2} \sim 10^{-12}$ is the sum of two positive terms one can safely assume that each $\left|\left(\Theta_{L}\right)_{N \mu}\right|^{2}$ and $\left|\left(\Theta_{L}\right)_{N e}\right|^{2} \sim 10^{-12}$ are individually smaller than $10^{-12}$.

TABLE IV. SM background processes at $100 \mathrm{TeV}$ and $30 \mathrm{ab}^{-1}$ for the trilepton signal $e^{+} e^{+} \mu^{-} \nu$ and $M_{W_{R}}=6 \mathrm{TeV}$, for two benchmark values of the heavy neutrino masses assuming $\left(\Theta_{L}\right)_{\mu N}=(\Theta)_{e N}=\frac{1}{\sqrt{2}}$. Backgrounds ending with $(j)$ were simulated with one matched jet. The charge misidentification probability has been taken from current ATLAS result from Ref. [57]. The jet to lepton fake rates for $t \bar{t}(j)$ and $Z / \gamma(j)$ have been taken as $10^{-4}$ universally. The NLO K-factor for backgrounds are taken from Ref. [63].

\begin{tabular}{|c|c|c|c|c|c|c|c|c|}
\hline \multirow[b]{2}{*}{$\underline{\sqrt{s}}=100 \mathrm{TeV}$} & \multicolumn{6}{|c|}{ Backgrounds } & \multicolumn{2}{|c|}{ Signal } \\
\hline & $t \bar{t} Z$ & $t \bar{t} W$ & $t \bar{t}(j)$ & $W Z(j)$ & $3 W(j)$ & $Z / \gamma(j)$ & $m_{N}(100 \mathrm{GeV})$ & $m_{N}(500 \mathrm{GeV})$ \\
\hline$e^{+} e^{+} \mu^{-}$(b-veto) & 199 & $1.1 \mathrm{~K}$ & $1.2 \mathrm{~K}$ & $9 \mathrm{~K}$ & 735 & $1.1 \mathrm{~K}$ & $1.9 \mathrm{M}$ & $1.8 \mathrm{M}$ \\
\hline$P_{T}$ cuts & 18.7 & 387 & 226 & $2.4 \mathrm{~K}$ & 254 & 244 & $1.34 \mathrm{M}$ & $1.30 \mathrm{M}$ \\
\hline $\mathscr{E}_{T}$ & 12.6 & 312 & 138 & $1.1 \mathrm{~K}$ & 165 & 18.7 & $1.1 \mathrm{M}$ & $1 \mathrm{M}$ \\
\hline$m_{\text {inv }}\left(e^{+} e^{+}\right)$cuts & 12.1 & 311 & 136 & 122 & 164 & 5.19 & $1.1 \mathrm{M}$ & $1 \mathrm{M}$ \\
\hline$m_{T}\left(e_{\mathrm{sub}}^{+} \mathscr{E}_{T}\right)$ & 4.42 & 116 & 65.1 & 22 & 85.9 & 0.344 & $1.1 \mathrm{M}$ & $0.99 \mathrm{M}$ \\
\hline$m_{\text {inv }}\left(e^{+} e^{+} \mu^{-} \mathscr{E}_{T}\right)$ & 0.126 & 7.60 & 5.82 & 0.336 & 9.72 & 0.0275 & $1 \mathrm{M}$ & $0.97 \mathrm{M}$ \\
\hline
\end{tabular}



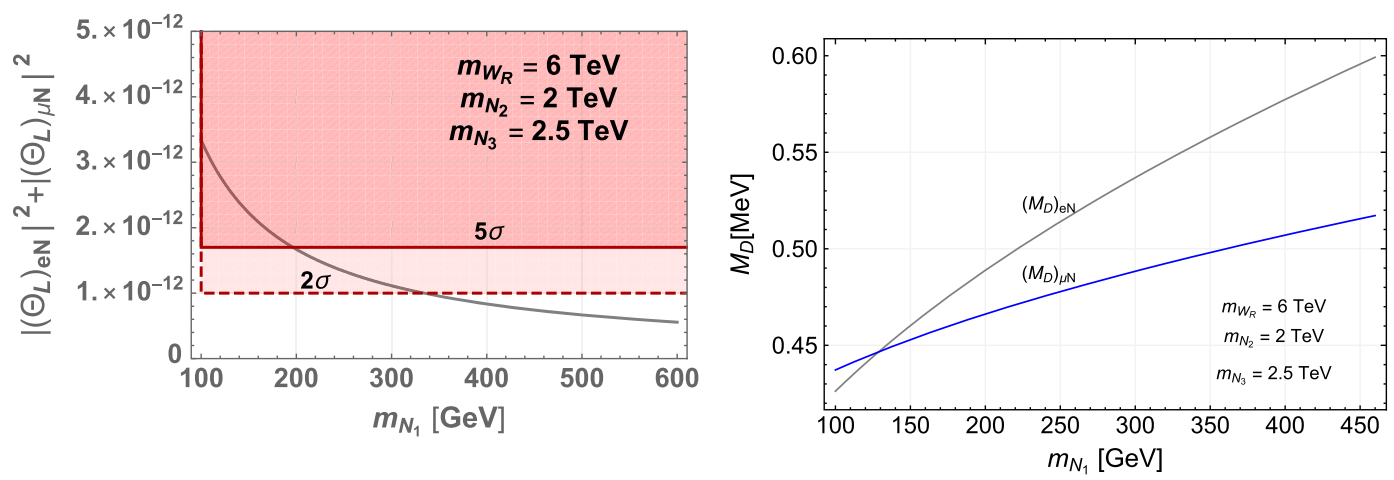

FIG. 10. Value for the heavy-light mixing angle combination $\left|\Theta_{N \mu}\right|^{2}+\left|\Theta_{N e}\right|^{2}$ (right) and the Dirac mass $M_{D}$ (left) as a function of the heavy neutrino mass, for $M_{W_{R}}=6 \mathrm{TeV}$ for a $2 \sigma$ exclusion region shown in Fig. 9 . We assume $V_{L}=V_{R}^{*}$ and the upper limit on light neutrino masses of $m_{\nu}=0.5 \mathrm{eV}$ [49].

In what follows, we discuss how the above estimates translate to the sensitivity for the Dirac mass matrix elements $\left(M_{D}\right)_{e N}$ and $\left(M_{D}\right)_{\mu N}$ at the $100 \mathrm{TeV} p p$ collider. To this end, it is instructive to show the relation between the Dirac mass matrix $M_{D}$ and the heavy, light neutrino mass matrices when $\left|\left(V_{L}\right)_{i, j}\right|=\left|\left(V_{R}\right)_{i, j}\right|$, since in this case the relation is simple enough to be written in a compact analytic form for both $\mathcal{C}$ and $\mathcal{P}$ cases. From Eq. (26) and for $\mathcal{C}$ as the LR symmetry, the Dirac mass matrix $M_{D}$ can be written as [15],

$$
M_{D}=V_{L}^{*} \hat{M}_{N} \sqrt{\frac{v_{L}}{v_{R}}-\frac{\hat{M}_{\nu}}{\hat{M}_{N}}} V_{L}^{\dagger} .
$$

Notice that this connection is lost for the nonminimal models, as can be explicitly seen in Eq. (33), since in this case there is an orthogonal, complex matrix $\mathcal{R}$ which makes the Dirac mass arbitrary.

The same considerations apply also for $\mathcal{P}$ as the LR symmetry where the Dirac mass matrix can be written as [16]

$$
M_{D}=V_{L} \hat{M}_{N} \sqrt{\frac{v_{L}}{v_{R}}-\frac{\hat{M}_{\nu}}{\hat{M}_{N}}} V_{L}^{\dagger}
$$

These results explicitly show that when $C P$ is an exact symmetry, both LR symmetries coincide. When $\left|\left(V_{L}\right)_{i, j}\right| \neq\left|\left(V_{R}\right)_{i, j}\right|$, the relation between the Dirac mass matrix and the heavy and light neutrino mass matrices shown in Eqs. (39) and (40) is far more complicated, and this general situation has been discussed in Refs. [15-17]. Nevertheless, even in this general case, the connection still exists and can be found by solving numerically some algebraic equations.

In short, the results we found apply for both parity and charge-conjugation as the LR symmetry. In Fig. 10 (right panel), we plot, using Eqs. (39) and (40), the values for
$\left(M_{D}\right)_{e N}$ and $\left(M_{D}\right)_{\mu N}$ in the same range of heavy neutrino masses. We can conclude that Dirac mass terms of the order of $10^{-1} \mathrm{MeV}$ can be probed at the $100 \mathrm{TeV} p p$ collider. In the case the results from the experiment does not match the predictions made by the minimal model, it would mean that the discrete LR symmetry must be explicitly broken in the Yukawa sector of the minimal model or that the LR symmetry is nonminimally realized.

Finally, it is interesting to observe that if approximate Parity symmetry is invoked as a solution to the strong $C P$ problem, the neutron electric dipole moment [66-69] constraint, together with the constraint coming from the indirect $C P$ violation $\left(\epsilon_{K}\right)$ in the kaon sector, would set $M_{W_{R}} \sim 20 \mathrm{TeV}$ [70]. This scale is the same scale that may be probed at the $100 \mathrm{TeV} p p$ collider. Hence, the $100 \mathrm{TeV}$ $p p$ collider offers the unique opportunity of finding the $W_{R}$ boson at the $20 \mathrm{TeV}$ scale, probing the Yukawa sector of the minimal model and offering a solution to the strong $C P$ problem by invoking parity as an approximate symmetry at higher energies [71].

\section{THE TRILEPTON SIGNAL IN OTHER BSM SCENARIOS}

The trilepton signal occurs in other beyond the Standard Model (BSM) scenarios, such as the MSSM (see for instance Ref. [72] and references therein), the inert Higgs doublet model and Little Higgs models. Therefore, a relevant question is how to distinguish the trilepton signal we study in this work from other BSM scenarios. For instance, in the MSSM the trilepton signal comes from the production via an on-shell $W$ boson of a chargino and the second lightest neutralino. Then, the chargino decays into one charged lepton and a sneutrino, where the sneutrino decays into a SM neutrino and the lightest stable neutralino. The second lightest neutralino decays into a charged lepton and a slepton, that then decays into another charged lepton and the lightest neutralino. As in the signal shown in Fig. 1, this signal may feature three charged leptons, but unlike our 
signal it has more sources of missing energy due to the two neutralinos in the final state. Furthermore, in the MSSM the final leptons might be predominantly tau and hence with final jets. This is because most models based on lepton universality predicts staus lighter than smuons and selectrons [72]. On top of the above differences, the pair of charged leptons coming from the neutralino are sameflavor, unlike the case studied in this work. This is also the case for the inert Higgs doublet model and the little Higgs models, since for those models there is always a sameflavor lepton pair coming from the decay of the $Z$ bosonsee for instance Refs. [73,74] for studies of the trilepton signal in the inert Higgs model and the little Higgs model respectively. These observations notwithstanding, a detailed, comparative treatment of the trilepton channel in various BSM scenarios (including the LRSM) and the method for distinguishing one scenario from another merits a dedicated future study.

\section{CONCLUSIONS}

In this work, we have analyzed the trilepton final state (produced via a $W_{R}$ gauge boson) as an effective channel for probing the Dirac mass terms of neutrinos at hadronic colliders in the context of left-right symmetric models. We have assessed the sensitivity to the heavy-light mixing of heavy neutrinos at the LHC, the HE-LHC and a $100 \mathrm{TeV}$ $p p$ collider using the $p p \rightarrow W_{R} \rightarrow e^{+} N \rightarrow e^{+}(N \rightarrow$ $e^{+} W^{-} \rightarrow e^{+} \mu^{-} \nu$ ) channel. Within the minimal framework, the relation between the Dirac mass matrix in terms of the heavy and light neutrino mass matrices implies that it is possible to translate the sensitivity to the heavy-light neutrino mixing into a bound on the neutrino Dirac mass $M_{D}$. For instance, we found that the minimal framework would not be seen at the LHC and the HE-LHC in the purely leptonic decays even with the ultimate integrated luminosities. Equivalently, this means that if any positive signal in the purely leptonic channel with the kinematic features described here is seen at the LHC, one would conclude left-right symmetry must be realized in a nonminimal context.

Finally, for a $100 \mathrm{TeV} p p$ collider with $30 \mathrm{ab}^{-1}$ of integrated luminosity, we have found that for $W_{R}$ boson masses between 6-20 $\mathrm{TeV}$ and heavy neutrinos masses between $80-460 \mathrm{GeV}$, the Dirac mass term $\left(M_{D}\right)_{e N}$ and $\left(M_{D}\right)_{\mu N}$ can be excluded at $2 \sigma$ up to masses of the order of $10^{-1} \mathrm{MeV}$ and when either parity or charge conjugation is the left-right symmetry. Furthermore, if the results of the experiment do not agree with the predictions given by the minimal model, the LR symmetry must be explicitly broken in the Yukawa sector or a nonminimal realization would be present.

\section{ACKNOWLEDGMENTS}

J. V. thanks Goran Senjanović for illuminating discussions and useful suggestions during all the stages of this work. J. C. H. thanks Martin Hirsch for useful discussions. J. V., J. C. H. and N. N. are grateful to the UMass Amherst Center for Fundamental Interactions for the hospitality, where they were visitors during the initial stages of this work. J. V. was funded by FONDECYT Grant No. 3170154 and by Conicyt PIA/Basal FB0821. J. C. H. is supported by Chile grants Fondecyt No. 1161463 and Conicyt PIA/ACT 1406. MJRM and H-L.L was supported in part under U.S. Department of Energy Contract No DESC0011095. N. N. was supported by FONDECYT (Chile) Grant No. 3170906. H.-L. L is also supported by the National Science Foundation of China under Grant No. 11875003.
[1] J. C. Pati and A. Salam, Lepton number as the fourth color, Phys. Rev. D 10, 275 (1974).

[2] R. N. Mohapatra and J.C. Pati, A natural left-right symmetry, Phys. Rev. D 11, 2558 (1975).

[3] G. Senjanović and R. N. Mohapatra, Exact left-right symmetry and spontaneous violation of parity, Phys. Rev. D 12, 1502 (1975).

[4] G. Senjanović, Spontaneous breakdown of parity in a class of gauge theories, Nucl. Phys. B153, 334 (1979).

[5] R. N. Mohapatra and G. Senjanović, Neutrino Mass and Spontaneous Parity Violation, Phys. Rev. Lett. 44, 912 (1980).

[6] R. N. Mohapatra and G. Senjanović, Neutrino masses and mixings in gauge models with spontaneous parity violation, Phys. Rev. D 23, 165 (1981).

[7] G. Aad et al. (ATLAS and CMS Collaborations), Combined Measurement of the Higgs Boson Mass in $p p$ Collisions at $\sqrt{s}=7$ and $8 \mathrm{TeV}$ with the ATLAS and CMS Experiments, Phys. Rev. Lett. 114, 191803 (2015).

[8] S. Chatrchyan et al. (CMS Collaboration), Evidence for the direct decay of the $125 \mathrm{GeV}$ Higgs boson to fermions, Nat. Phys. 10, 557 (2014).

[9] P. Minkowski, $\mu \rightarrow e \gamma$ at a rate of one out of $10^{9}$ muon decays?, Phys. Lett. 67B, 421 (1977).

[10] S. L. Glashow, The future of elementary particle physics, NATO Sci. Ser. B 61, 687 (1980).

[11] M. Gell-Mann, P. Ramond, and R. Slansky, Complex spinors and unified theories, Conf. Proc. C790927, 315 (1979).

[12] T. Yanagida, Horizontal symmetry and masses of neutrinos, Conf. Proc. C7902131, 95 (1979).

[13] J. Schechter and J. W. F. Valle, Neutrino masses in $\mathrm{SU}(2) \times$ U(1) theories, Phys. Rev. D 22, 2227 (1980). 
[14] W.-Y. Keung and G. Senjanović, Majorana Neutrinos and the Production of the Right-Handed Charged Gauge Boson, Phys. Rev. Lett. 50, 1427 (1983).

[15] M. Nemevsek, G. Senjanović, and V. Tello, Connecting Dirac and Majorana Neutrino Mass Matrices in the Minimal Left-Right Symmetric Model, Phys. Rev. Lett. 110, 151802 (2013).

[16] G. Senjanović and V. Tello, Probing Seesaw with Parity Restoration, Phys. Rev. Lett. 119, 201803 (2017).

[17] G. Senjanovic and V. Tello, Disentangling seesaw in the minimal left-right symmetric model, arXiv:1812.03790.

[18] J.A. Casas and A. Ibarra, Oscillating neutrinos and $\mu \rightarrow e, \gamma$, Nucl. Phys. B618, 171 (2001).

[19] A. Ferrari, J. Collot, M.-L. Andrieux, B. Belhorma, P. de Saintignon, J.-Y. Hostachy, Ph. Martin, and M. Wielers, Sensitivity study for new gauge bosons and right-handed majorana neutrinos in $p p$ collisions at $\sqrt{s}=14 \mathrm{TeV}$, Phys. Rev. D 62, 013001 (2000).

[20] T. Han, I. Lewis, R. Ruiz, and Z.-g. Si, Lepton number violation and $W^{\prime}$ chiral couplings at the LHC, Phys. Rev. D 87, 035011 (2013).

[21] E. Izaguirre and B. Shuve, Multilepton and lepton jet probes of sub-weak-scale right-handed neutrinos, Phys. Rev. D 91, 093010 (2015).

[22] A. M. Sirunyan et al. (CMS Collaboration), Search for Heavy Neutral Leptons in Events with Three Charged Leptons in Proton-Proton Collisions at $\sqrt{s}=13 \mathrm{TeV}$, Phys. Rev. Lett. 120, 221801 (2018).

[23] F. del Aguila, J. A. Aguilar-Saavedra, and J. de Blas, Trilepton signals: The golden channel for seesaw searches at LHC, Acta Phys. Pol. B 40, 2901 (2009).

[24] A. Das, N. Nagata, and N. Okada, Testing the 2-TeV resonance with trileptons, J. High Energy Phys. 03 (2016) 049.

[25] J. Kersten and A. Yu. Smirnov, Right-handed neutrinos at CERN LHC and the mechanism of neutrino mass generation, Phys. Rev. D 76, 073005 (2007).

[26] A. de Gouvea, GeV seesaw, accidentally small neutrino masses, and Higgs decays to neutrinos, arXiv:0706.1732.

[27] Z.-z. Xing, Naturalness and testability of $\mathrm{TeV}$ seesaw mechanisms, Prog. Theor. Phys. Suppl. 180, 112 (2009).

[28] X.-G. He, S. Oh, J. Tandean, and C.-C. Wen, Large mixing of light and heavy neutrinos in seesaw models and the LHC, Phys. Rev. D 80, 073012 (2009).

[29] A. Ibarra, E. Molinaro, and S. T. Petcov, TeV scale see-saw mechanisms of neutrino mass generation, the Majorana nature of the heavy singlet neutrinos and $(\beta \beta)_{0 \nu}$-decay, J. High Energy Phys. 09 (2010) 108.

[30] N. Haba, T. Horita, K. Kaneta, and Y. Mimura, TeV-scale seesaw with non-negligible left-right neutrino mixings, arXiv:1110.2252.

[31] M. Mitra, G. Senjanovic, and F. Vissani, Neutrinoless double beta decay and heavy sterile neutrinos, Nucl. Phys. B856, 26 (2012).

[32] C.-Y. Chen, P. S. B. Dev, and R. N. Mohapatra, Probing heavy-light neutrino mixing in left-right seesaw models at the LHC, Phys. Rev. D 88, 033014 (2013).

[33] E. K. Akhmedov and M. Frigerio, Interplay of type I and type II seesaw contributions to neutrino mass, J. High Energy Phys. 01 (2007) 043.
[34] E. K. Akhmedov, M. Blennow, T. Hallgren, T. Konstandin, and T. Ohlsson, Stability and leptogenesis in the left-right symmetric seesaw mechanism, J. High Energy Phys. 04 (2007) 022.

[35] W. Chao, S. Luo, Z.-z. Xing, and S. Zhou, A compromise between neutrino masses and collider signatures in the typeII seesaw model, Phys. Rev. D 77, 016001 (2008).

[36] G. Senjanovi and V. Tello, Right Handed Quark Mixing in Left-Right Symmetric Theory, Phys. Rev. Lett. 114, 071801 (2015).

[37] G. Senjanovi and V. Tello, Restoration of parity and the right-handed analog of the CKM matrix, Phys. Rev. D 94, 095023 (2016).

[38] S. Dube, D. Gadkari, and A. M. Thalapillil, Lepton-jets and low-mass sterile neutrinos at hadron colliders, Phys. Rev. D 96, 055031 (2017).

[39] V. Tello, Connections between the high and low energy violation of lepton and flavor numbers in the minimal leftright symmetric model, Ph.D. thesis, SISSA, Trieste, 2012.

[40] A. Maiezza, G. Senjanović, and J. C. Vasquez, Higgs sector of the minimal left-right symmetric theory, Phys. Rev. D 95, 095004 (2017).

[41] M. K. Parida and A. Raychaudhuri, Inverse see-saw, leptogenesis, observable proton decay and $\Delta_{\mathrm{R}}^{ \pm \pm}$in SUSY SO(10) with heavy $W_{R}$, Phys. Rev. D 82, 093017 (2010).

[42] C. Arbelez, M. Hirsch, M. Malinsk, and J. C. Romo, LHCscale left-right symmetry and unification, Phys. Rev. D 89, 035002 (2014).

[43] R. N. Mohapatra and J. W. F. Valle, Neutrino mass and baryon number nonconservation in superstring models, Phys. Rev. D 34, 1642 (1986).

[44] P. S. Bhupal Dev and R. N. Mohapatra, Unified Explanation of the eejj, Diboson and Dijet Resonances at the LHC, Phys. Rev. Lett. 115, 181803 (2015).

[45] G. Anamiati, M. Hirsch, and E. Nardi, Quasi-Dirac neutrinos at the LHC, J. High Energy Phys. 10 (2016) 010.

[46] G. Anamiati, R. M. Fonseca, and M. Hirsch, Quasi dirac neutrino oscillations, Phys. Rev. D 97, 095008 (2018).

[47] M. Mitra, R. Ruiz, D. J. Scott, and M. Spannowsky, Neutrino jets from high-mass $W_{R}$ gauge bosons in TeVscale left-right symmetric models, Phys. Rev. D 94, 095016 (2016).

[48] M. Nemevsek, F. Nesti, and G. Popara, Keung-Senjanović process at LHC: From LNV to displaced vertices to invisible decays, Phys. Rev. D 97, 115018 (2018).

[49] K. Nakamura et al. (Particle Data Group), Review of particle physics, J. Phys. G 37, 075021 (2010).

[50] C. Arbelaz, C. Dib, I. Schmidt, and J. C. Vasquez, Probing the Dirac or Majorana nature of the heavy neutrinos in pure leptonic decays at the LHC, Phys. Rev. D 97, 055011 (2018).

[51] A. Alloul, N. D. Christensen, C. Degrande, C. Duhr, and B. Fuks, FeynRules 2.0: A complete toolbox for treelevel phenomenology, Comput. Phys. Commun. 185, 2250 (2014).

[52] A. Roitgrund, G. Eilam, and S. Bar-Shalom, Implementation of the left-right symmetric model in FeynRules, Comput. Phys. Commun. 203, 18 (2016).

[53] M. Nemevsek, F. Nesti, and J. C. Vasquez, Majorana Higgses at colliders, J. High Energy Phys. 04 (2017) 114. 
[54] J. Alwall, R. Frederix, S. Frixione, V. Hirschi, F. Maltoni, O. Mattelaer, H.-S. Shao, T. Stelzer, P. Torrielli, and M. Zaro, The automated computation of tree-level and next-toleading order differential cross sections, and their matching to parton shower simulations, J. High Energy Phys. 07 (2014) 079.

[55] T. Sjostrand, S. Mrenna, and P.Z. Skands, PYTHIA 6.4 physics and manual, J. High Energy Phys. 05 (2006) 026.

[56] J. de Favereau, C. Delaere, P. Demin, A. Giammanco, V. Lematre, A. Mertens, and M. Selvaggi (DELPHES 3 Collaboration), DELPHES 3: A modular framework for fast simulation of a generic collider experiment, J. High Energy Phys. 02 (2014) 057.

[57] M. Aaboud et al. (ATLAS Collaboration), Search for doubly charged Higgs boson production in multi-lepton final states with the ATLAS detector using protonproton collisions at $\sqrt{s}=13$ TeV, Eur. Phys. J. C 78, 199 (2018).

[58] M. Nemevsek, F. Nesti, G. Senjanović, and Y. Zhang, First limits on left-right symmetry scale from LHC data, Phys. Rev. D 83, 115014 (2011).

[59] J. C. Helo, M. Hirsch, and S. Kovalenko, Heavy neutrino searches at the LHC with displaced vertices, Phys. Rev. D 89, 073005 (2014).

[60] G. Cottin, J. C. Helo, and M. Hirsch, Searches for light sterile neutrinos with multitrack displaced vertices, Phys. Rev. D 97, 055025 (2018).

[61] G. Cottin, J. C. Helo, and M. Hirsch, Displaced vertices as probes of sterile neutrino mixing at the LHC, Phys. Rev. D 98, 035012 (2018).

[62] A. Das, P. S. B. Dev, and R. N. Mohapatra, Same sign versus opposite sign dileptons as a probe of low scale seesaw mechanisms, Phys. Rev. D 97, 015018 (2018).

[63] M. L. Mangano et al., Physics at a $100 \mathrm{TeV}$ pp collider: Standard model processes, arXiv:1607.01831.
[64] M. Nemevsek, G. Senjanovic, and Y. Zhang, Warm dark matter in low scale left-right theory, J. Cosmol. Astropart. Phys. 07 (2012) 006.

[65] M. Aaboud et al. (ATLAS Collaboration), Search for heavy Majorana or Dirac neutrinos and right-handed $W$ gauge bosons in final states with two charged leptons and two jets at $\sqrt{s}=13 \mathrm{TeV}$ with the ATLAS detector, J. High Energy Phys. 01 (2019) 016.

[66] J. S. M. Ginges and V. V. Flambaum, Violations of fundamental symmetries in atoms and tests of unification theories of elementary particles, Phys. Rep. 397, 63 (2004).

[67] M. Pospelov and A. Ritz, Electric dipole moments as probes of new physics, Ann. Phys. (Amsterdam) 318, 119 (2005).

[68] T. Fukuyama, Searching for new physics beyond the Standard Model in electric dipole moment, Int. J. Mod. Phys. A 27, 1230015 (2012).

[69] J. Engel, M. J. Ramsey-Musolf, and U. van Kolck, Electric dipole moments of nucleons, nuclei, and atoms: The Standard Model and beyond, Prog. Part. Nucl. Phys. 71, 21 (2013).

[70] A. Maiezza and M. Nemevsek, Strong P invariance, neutron electric dipole moment, and minimal left-right parity at LHC, Phys. Rev. D 90, 095002 (2014).

[71] R. N. Mohapatra and G. Senjanović, Natural suppression of strong p and t noninvariance, Phys. Lett. 79B, 283 (1978).

[72] S. P. Martin, A supersymmetry primer, Adv. Ser. Dir. High Energy Phys. 21, 1 (2010); 18, 1 (1998).

[73] X. Miao, S. Su, and B. Thomas, Trilepton signals in the inert doublet model, Phys. Rev. D 82, 035009 (2010).

[74] A. Datta, P. Dey, S. K. Gupta, B. Mukhopadhyaya, and A. Nyffeler, Distinguishing the littlest Higgs model with T-parity from supersymmetry at the LHC using trileptons, Phys. Lett. B 659, 308 (2008). 